\title{
A Test Statistic in the Complex Wishart Distribution and Its Application to Change Detection in Polarimetric SAR Data
}

\author{
Knut Conradsen, Allan Aasbjerg Nielsen, Jesper Schou, and Henning Skriver
}

\begin{abstract}
When working with multilook fully polarimetric synthetic aperture radar (SAR) data, an appropriate way of representing the backscattered signal consists of the so-called covariance matrix. For each pixel, this is a $3 \times 3$ Hermitian positive definite matrix that follows a complex Wishart distribution. Based on this distribution, a test statistic for equality of two such matrices and an associated asymptotic probability for obtaining a smaller value of the test statistic are derived and applied successfully to change detection in polarimetric SAR data. In a case study, EMISAR L-band data from April 17, 1998 and May 20, 1998 covering agricultural fields near Foulum, Denmark are used. Multilook full covariance matrix data, azimuthal symmetric data, covariance matrix diagonal-only data, and horizontal-horizontal (HH), vertical-vertical (VV), or horizontal-vertical (HV) data alone can be used. If applied to $\mathrm{HH}, \mathrm{VV}$, or $\mathrm{HV}$ data alone, the derived test statistic reduces to the well-known gamma likelihood-ratio test statistic. The derived test statistic and the associated significance value can be applied as a line or edge detector in fully polarimetric SAR data also.
\end{abstract}

Index Terms-Covariance matrix test statistic, EMISAR, radar applications, radar polarimetry, remote sensing change detection.

\section{INTRODUCTION}

D UE TO ITS all-weather mapping capability independently of, for instance, cloud cover, synthetic aperture radar (SAR) data hold a strong potential, for example, for change detection studies in remote sensing applications. In this paper, multitemporal SAR images of agricultural fields are used to demonstrate a new change detection method for polarimetric SAR data. It is well known that the development of different crops over time causes changes in the backscatter. The radar backscattering is sensitive to the dielectric properties of the vegetation and the soil, to the plant structure (i.e., the size, shape, and orientation distributions of the scatterers), to the surface roughness, and to the canopy structure (e.g., row direction and spacing and cover fraction) [1], [2].

The polarimetric SAR measures the amplitude and phase of backscattered signals in four combinations of the linear receive and transmit polarizations: horizontal-horizontal $(\mathrm{HH})$,

Manuscript received June 8, 2001; revised October 17, 2002. This work was supported in part by The Danish National Research Councils in part under the Earth Observation Programme and in part under the European Space Agency Follow-On Research Programme. The EMISAR data acquisitions and part of the data processing were supported by the Danish National Research Foundation.

K. Conradsen and A. A. Nielsen are with IMM, Informatics and Mathematical Modelling, Technical University of Denmark, DK-2800 Lyngby, Denmark (e-mail: aa@imm.dtu.dk).

J. Schou and H. Skriver are with EMI, Section of Electromagnetic Systems, Technical University of Denmark (Ørsted DTU), DK-2800 Lyngby, Denmark. Digital Object Identifier 10.1109/TGRS.2002.808066 horizontal-vertical (HV), vertical-horizontal (VH), and vertical-vertical (VV). These signals form the complex scattering matrix that relates the incident and the scattered electric fields [3]. The inherent speckle in the SAR data can be reduced by spatial averaging at the expense of loss of spatial resolution. In this so-called multilook case, a more appropriate representation of the backscattered signal is the covariance matrix in which the average properties of a group of resolution cells can be expressed in a single matrix. The average covariance matrix is defined as [3]

$$
\langle C\rangle=\left[\begin{array}{lll}
\left\langle S_{\mathrm{hh}} S_{\mathrm{hh}}^{*}\right\rangle & \left\langle S_{\mathrm{hh}} S_{\mathrm{hv}}^{*}\right\rangle & \left\langle S_{\mathrm{hh}} S_{\mathrm{vv}}^{*}\right\rangle \\
\left\langle S_{\mathrm{hv}} S_{\mathrm{hh}}^{*}\right\rangle & \left\langle S_{\mathrm{hv}} S_{\mathrm{hv}}^{*}\right\rangle & \left\langle S_{\mathrm{hv}} S_{\mathrm{vv}}^{*}\right\rangle \\
\left\langle S_{\mathrm{vv}} S_{\mathrm{hh}}^{*}\right\rangle & \left\langle S_{\mathrm{vv}} S_{\mathrm{hv}}^{*}\right\rangle & \left\langle S_{\mathrm{vv}} S_{\mathrm{vv}}^{*}\right\rangle
\end{array}\right]
$$

where $\langle\cdot\rangle$ denotes ensemble averaging; ${ }^{*}$ denotes complex conjugation; and $S_{r t}$ is the complex scattering amplitude for receive polarization $r$ and transmit polarization $t$ ( $r$ and $t$ are either $h$ for horizontal or $v$ for vertical). Reciprocity, which normally applies to natural targets, gives $S_{\mathrm{hv}}=S_{\mathrm{vh}}$ (in the backscattering direction using the backscattering alignment convention [3]) and results in the covariance matrix (1) with rank 3. $\langle C\rangle$ follows a complex Wishart distribution [4]. The components in the covariance matrix containing both co- and cross-polarized scattering matrix elements often contain little information. For randomly distributed targets with azimuthal symmetry, the elements are zero [5].

In this paper, a test statistic for equality of two complex covariance matrices and an associated asymptotic probability measure for obtaining a smaller value of the test statistic are derived and applied to change detection in fully polarimetric SAR data. In [6], a change detection scheme based on canonical correlations analysis is applied to scalar EMISAR data (see also [7]-[10]).

If used with $\mathrm{HH}, \mathrm{VV}$, or $\mathrm{HV}$ data only, the test statistic reduces to the well-known test statistic for equality of the scale parameters in two gamma distributions.

The derived test statistic and the associated significance measure can be applied as a line or edge detector in fully polarimetric SAR data also [11].

Section II sketches important aspects of the complex Gaussian and Wishart distributions, the likelihood-ratio test statistic in the complex Wishart distribution, and the associated significance measure. Section III gives a case study in which data from the Danish airborne EMISAR [12], [13] are used. Section IV discusses the results from the case study, and Section V concludes. 


\section{THEORY}

This section describes the complex normal and Wishart distributions and the likelihood-ratio test for equality of two complex Wishart matrices. For a more thorough description, see the Appendix.

\section{A. Complex Normal Distribution}

We say that a $p$-dimensional random complex vector $\boldsymbol{Z}$ follows a complex multivariate normal distribution with mean $\mathbf{0}$ and dispersion matrix $\boldsymbol{\Sigma}$, i.e.,

$$
\boldsymbol{Z}=\left[Z_{1} \cdots Z_{p}\right]^{T} \in N_{C}(\mathbf{0}, \mathbf{\Sigma})
$$

if the frequency function is

$$
\begin{aligned}
f(z) & =\frac{1}{\pi^{p}|\boldsymbol{\Sigma}|} \exp \left\{-z^{* T} \boldsymbol{\Sigma}^{-1} z\right\} \\
& =\frac{1}{\pi^{p}|\boldsymbol{\Sigma}|} \exp \left\{-\operatorname{tr}\left[\boldsymbol{\Sigma}^{-1} z z^{* T}\right]\right\}
\end{aligned}
$$

where $|\cdot|$ denotes the determinant; tr denotes the trace of a matrix; and ${ }^{* T}$ denotes complex conjugation $\left({ }^{*}\right)$ and transpose $\left({ }^{T}\right)$.

\section{B. Complex Wishart Distribution}

We say that a Hermitian positive definite random $p \times p$ matrix $\boldsymbol{X}$ follows a complex Wishart distribution, i.e.,

$$
\boldsymbol{X} \in W_{C}(p, n, \boldsymbol{\Sigma})
$$

if the frequency function is

$$
w(\boldsymbol{x})=\frac{1}{\Gamma_{p}(n)} \frac{1}{|\boldsymbol{\Sigma}|^{n}}|\boldsymbol{x}|^{n-p} \exp \left\{-\operatorname{tr}\left[\boldsymbol{\Sigma}^{-1} \boldsymbol{w}\right]\right\}
$$

where

$$
\Gamma_{p}(n)=\pi^{p(p-1) / 2} \prod_{j=1}^{p} \Gamma(n-j+1) .
$$

The frequency function is defined for $\boldsymbol{w}$ positive definite.

If $\boldsymbol{X}$ and $\boldsymbol{Y}$ are independent and both follow complex Wishart distributions

$$
\boldsymbol{X} \in W_{C}(p, n, \boldsymbol{\Sigma}) \text { and } \boldsymbol{Y} \in W_{C}(p, m, \boldsymbol{\Sigma})
$$

then their sum also follows a complex Wishart distribution

$$
\boldsymbol{S}=\boldsymbol{X}+\boldsymbol{Y} \in W_{C}(p, n+m, \boldsymbol{\Sigma}) .
$$

\section{Test for Equality of Two Complex Wishart Matrices}

Let the independent $p \times p$ Hermitian positive definite matrices $X$ and $Y$ be complex Wishart distributed, i.e., $\boldsymbol{X} \in W_{C}\left(p, n, \boldsymbol{\Sigma}_{x}\right)$ with $\hat{\boldsymbol{\Sigma}}_{x}=1 / n \boldsymbol{X}$ and $\boldsymbol{Y} \in W_{C}\left(p, m, \boldsymbol{\Sigma}_{y}\right)$ with $\hat{\boldsymbol{\Sigma}}_{y}=1 / m \boldsymbol{Y}$. We consider the null hypothesis $H_{0}: \boldsymbol{\Sigma}_{x}=\boldsymbol{\Sigma}_{y}$, which states that the two matrices are equal against the alternative hypothesis $H_{1}: \boldsymbol{\Sigma}_{x} \neq \boldsymbol{\Sigma}_{y}$.

In general, suppose that the observations on which we shall base our test have joint density $f(x, \theta)$ where $\theta$ is the set of parameters of the probability function that has generated the data. Then $H_{0}$ states that $\theta \in \omega_{0}$ where $\omega_{0}$ is a subset of the set $\Omega$ of all possible $\theta$. $H_{1}$ states that $\omega \in \omega_{1}$ where $\omega_{0}$ and $\omega_{1}$ are disjoint, and often $\Omega=\omega_{0} \cup \omega_{1}$. The likelihood ratio

$$
Q=\frac{\max _{\theta \in \omega_{0}} L(\theta)}{\max _{\theta \in \Omega} L(\theta)}
$$

where $L$ is the likelihood function rejects $H_{0}$ for small values.

If $H_{0}$ is true (in statistical parlance "under $H_{0}$ "), then in our case $\boldsymbol{S}=\boldsymbol{X}+\boldsymbol{Y} \in W_{C}(p, n+m, \boldsymbol{\Sigma})$ with $\hat{\boldsymbol{\Sigma}}=1 /(n+m) \boldsymbol{S}=$ $1 /(n+m)(\boldsymbol{X}+\boldsymbol{Y})$. The likelihood-ratio test statistic becomes

$$
Q=\frac{L(\hat{\boldsymbol{\Sigma}})}{L_{x}\left(\hat{\boldsymbol{\Sigma}}_{x}\right) L_{y}\left(\hat{\boldsymbol{\Sigma}}_{y}\right)} .
$$

Here

$$
L_{x}\left(\boldsymbol{\Sigma}_{x}\right)=\frac{1}{\Gamma_{p}(n)}\left|\boldsymbol{\Sigma}_{x}\right|^{-n}|\boldsymbol{X}|^{n-p} \exp \left\{-\operatorname{tr}\left[\boldsymbol{\Sigma}_{x}^{-1} \boldsymbol{X}\right]\right\}
$$

and

$$
L_{x}\left(\hat{\boldsymbol{\Sigma}}_{x}\right)=\frac{1}{\Gamma_{p}(n)}\left|\frac{1}{n} \boldsymbol{X}\right|^{-n}|\boldsymbol{X}|^{n-p} \exp \{-n \operatorname{tr} \boldsymbol{I}\}
$$

where $\boldsymbol{I}$ is the identity matrix $(\operatorname{tr} \boldsymbol{I}=p)$. Similar expressions are valid for $L_{y}\left(\boldsymbol{\Sigma}_{y}\right)$ and $L_{y}\left(\hat{\boldsymbol{\Sigma}}_{y}\right)$. For the numerator of $Q$ we get

$$
\begin{aligned}
L(\boldsymbol{\Sigma})=\frac{1}{\Gamma_{p}(n) \Gamma_{p}(m)}|\boldsymbol{\Sigma}|^{-(n+m)}|\boldsymbol{X}|^{n-p}|\boldsymbol{Y}|^{m-p} \\
\cdot \exp \left\{-\operatorname{tr}\left[\boldsymbol{\Sigma}^{-1}(\boldsymbol{X}+\boldsymbol{Y})\right]\right\}
\end{aligned}
$$

and

$$
\begin{aligned}
L(\hat{\boldsymbol{\Sigma}})=\frac{1}{\Gamma_{p}(n) \Gamma_{p}(m)}\left|\frac{1}{n+m}(\boldsymbol{X}+\boldsymbol{Y})\right|^{-(n+m)} \\
\cdot|\boldsymbol{X}|^{n-p}|\boldsymbol{Y}|^{m-p} \exp \{-(n+m) \operatorname{tr} \boldsymbol{I}\} .
\end{aligned}
$$

This leads to the desired likelihood-ratio test statistic

$$
Q=\frac{(n+m)^{p(n+m)}}{n^{p n} m^{p m}} \frac{|\boldsymbol{X}|^{n}|\boldsymbol{Y}|^{m}}{|\boldsymbol{X}+\boldsymbol{Y}|^{n+m}} .
$$

If $n=m$, which is typically the case for change detection, we get

$$
\ln Q=n(2 p \ln 2+\ln |\boldsymbol{X}|+\ln |\boldsymbol{Y}|-2 \ln |\boldsymbol{X}+\boldsymbol{Y}|) .
$$

If

$$
\rho=1-\frac{2 p^{2}-1}{6 p}\left(\frac{1}{n}+\frac{1}{m}-\frac{1}{n+m}\right)
$$

and

$$
\begin{aligned}
\omega_{2}=-\frac{p^{2}}{4}\left(1-\frac{1}{\rho}\right)^{2} & +\frac{p^{2}\left(p^{2}-1\right)}{24} \\
& \cdot\left(\frac{1}{n^{2}}+\frac{1}{m^{2}}-\frac{1}{(n+m)^{2}}\right) \frac{1}{\rho^{2}}
\end{aligned}
$$

then the probability of finding a smaller value of $-2 \rho \ln Q$ is

$$
\begin{aligned}
& P\{-2 \rho \ln Q \leq z\} \simeq P\left\{\chi^{2}\left(p^{2}\right) \leq z\right\} \\
& \quad+\omega_{2}\left[P\left\{\chi^{2}\left(p^{2}+4\right) \leq z\right\}-P\left\{\chi^{2}\left(p^{2}\right) \leq z\right\}\right] .
\end{aligned}
$$


For covariance matrix data, $p=3$. For HH, HV, or VV data, $p=1$. In the latter case, $\boldsymbol{X}$ and $\boldsymbol{Y}$ are therefore scalars $X$ and $Y$, and $Q$ reduces to

$$
Q=\frac{(n+m)^{n+m}}{n^{n} m^{m}} \frac{X^{n} Y^{m}}{(X+Y)^{n+m}}
$$

which is equivalent to the well-known likelihood-ratio test statistic for the equality of two gamma parameters [14], [15] (see the Appendix).

Fig. 1 shows $\rho$ and $\omega_{2}$ as functions of the number of looks for $n=m$ and $p=3$.

\section{Azimuthal Symmetry}

By swapping first rows and then columns two and three in $\langle C\rangle$ in (1), we obtain in the azimuthal symmetry case

$$
\left[\begin{array}{ccc}
\left\langle S_{\mathrm{hh}} S_{\mathrm{hh}}^{*}\right\rangle & \left\langle S_{\mathrm{hh}} S_{\mathrm{vv}}^{*}\right\rangle & 0 \\
\left\langle S_{\mathrm{vv}} S_{\mathrm{hh}}^{*}\right\rangle & \left\langle S_{\mathrm{vv}} S_{\mathrm{vv}}^{*}\right\rangle & 0 \\
0 & 0 & \left\langle S_{\mathrm{hv}} S_{\mathrm{hv}}^{*}\right\rangle
\end{array}\right]=\left[\begin{array}{cc}
\boldsymbol{X}_{1} & 0 \\
0 & \boldsymbol{X}_{2}
\end{array}\right]=\boldsymbol{X}
$$

where $\boldsymbol{X}_{1}$ is $p_{1} \times p_{1}$ (here $2 \times 2$ ), and $\boldsymbol{X}_{2}=\left\langle S_{\mathrm{hv}} S_{\mathrm{hv}}^{*}\right\rangle$ is $p_{2} \times p_{2}$ (here $1 \times 1$ ). This matrix is not Wishart distributed. We now consider $\boldsymbol{X}_{1} \in W_{C}\left(p_{1}, n, \boldsymbol{\Sigma}_{x 1}\right), \boldsymbol{X}_{2} \in W_{C}\left(p_{2}, n, \boldsymbol{\Sigma}_{x 2}\right), \boldsymbol{Y}_{1} \in$ $W_{C}\left(p_{1}, m, \boldsymbol{\Sigma}_{y 1}\right)$, and $\boldsymbol{Y}_{2} \in W_{C}\left(p_{2}, m, \boldsymbol{\Sigma}_{y 2}\right)$, and we assume that $\boldsymbol{X}_{1}, \boldsymbol{X}_{2}, \boldsymbol{Y}_{1}$, and $\boldsymbol{Y}_{2}$ are mutually independent.

We want to test the hypothesis $H_{0}: \boldsymbol{\Sigma}_{x 1}=\boldsymbol{\Sigma}_{y 1}\left(=\boldsymbol{\Sigma}_{1}\right)$ and $\boldsymbol{\Sigma}_{x 2}=\boldsymbol{\Sigma}_{y 2}\left(=\boldsymbol{\Sigma}_{2}\right)$ against all alternatives. We have the likelihood function

$$
\begin{aligned}
& L\left(\boldsymbol{\Sigma}_{x 1}, \boldsymbol{\Sigma}_{x 2}, \boldsymbol{\Sigma}_{y 1}, \boldsymbol{\Sigma}_{y 2}\right) \\
& \quad=L^{(1)}\left(\boldsymbol{\Sigma}_{x 1}\right) L^{(2)}\left(\boldsymbol{\Sigma}_{x 2}\right) L^{(3)}\left(\boldsymbol{\Sigma}_{y 1}\right) L^{(4)}\left(\boldsymbol{\Sigma}_{y 2}\right) .
\end{aligned}
$$

The likelihood-ratio test statistic becomes

$$
\begin{aligned}
Q & =\frac{(n+m)^{p(n+m)}}{n^{p n} m^{p m}} \frac{\left|\boldsymbol{X}_{1}\right|^{n}\left|\boldsymbol{Y}_{1}\right|^{m}\left|\boldsymbol{X}_{2}\right|^{n}\left|\boldsymbol{Y}_{2}\right|^{m}}{\left|\boldsymbol{X}_{1}+\boldsymbol{Y}_{1}\right|^{n+m}\left|\boldsymbol{X}_{2}+\boldsymbol{Y}_{2}\right|^{n+m}} \\
& =\frac{(n+m)^{p(n+m)}}{n^{p n} m^{p m}} \frac{|\boldsymbol{X}|^{n}|\boldsymbol{Y}|^{m}}{|\boldsymbol{X}+\boldsymbol{Y}|^{n+m}}
\end{aligned}
$$

where the latter equality is due to the fact that the determinant of a block diagonal matrix is the product of the determinants of the diagonal elements, i.e., we get the same test statistic as in the full covariance matrix case. In this case $p^{2}=p_{1}^{2}+p_{2}^{2}$. If

$$
\begin{aligned}
\rho & =\frac{1}{p^{2}}\left(p_{1}^{2} \rho_{1}+p_{2}^{2} \rho_{2}\right) \\
\rho_{i} & =1-\frac{2 p_{i}^{2}-1}{6 p_{i}}\left(\frac{1}{n}+\frac{1}{m}-\frac{1}{n+m}\right)
\end{aligned}
$$

and

$$
\begin{aligned}
\omega_{2}=-\frac{p^{2}}{4}\left(1-\frac{1}{\rho}\right)^{2} & +\frac{p_{1}^{2}\left(p_{1}^{2}-1\right)+p_{2}^{2}\left(p_{2}^{2}-1\right)}{24} \\
& \cdot\left(\frac{1}{n^{2}}+\frac{1}{m^{2}}-\frac{1}{(n+m)^{2}}\right) \frac{1}{\rho^{2}}
\end{aligned}
$$

then the probability of finding a smaller value of $-2 \rho \ln Q$ is

$$
\begin{aligned}
& P\{-2 \rho \ln Q \leq z\} \simeq P\left\{\chi^{2}\left(p^{2}\right) \leq z\right\} \\
& \quad+\omega_{2}\left[P\left\{\chi^{2}\left(p^{2}+4\right) \leq z\right\}-P\left\{\chi^{2}\left(p^{2}\right) \leq z\right\}\right] .
\end{aligned}
$$
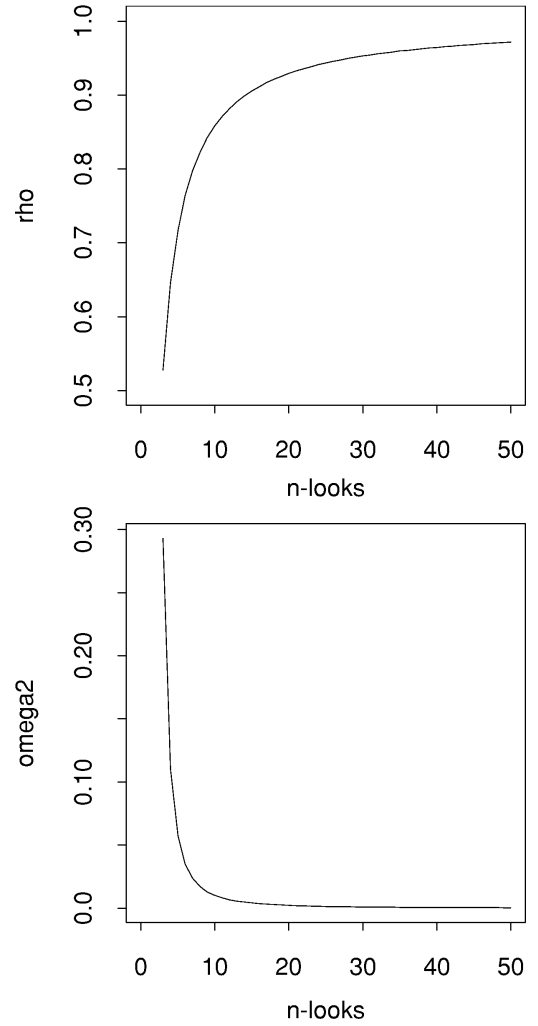

Fig. 1. $\quad \rho$ and $\omega_{2}$ as functions of the number of looks for $n=m$ and $p=3$.

\section{DATA}

To illustrate the change detection capability of the derived test statistic, EMISAR and ground data from an agricultural test site at the Research Center Foulum located in Central Jutland, Denmark are used. Agricultural fields have been selected for the analysis because of the large change in the polarimetric properties for such areas, due to the development of the crops with time. Polarimetric parameters of agricultural crops have previously been analyzed from this area [2].

\section{A. SAR Data and Calibration}

The EMISAR system is a fully polarimetric airborne SAR system, and it operates at two frequencies: C-band (5.3-GHz/5.7-cm wavelength) and L-band $(1.25-\mathrm{GHz} / 24-\mathrm{cm}$ wavelength) [11], [12]. The SAR system is normally operated from an altitude of approximately $12500 \mathrm{~m}$; the spatial resolution is $2 \mathrm{~m} \times 2 \mathrm{~m}$ (one-look); the ground range swath is approximately $12 \mathrm{~km}$; and typical incidence angles range from $35^{\circ}$ to $60^{\circ}$. The processed data from this system are fully calibrated by means of an advanced internal calibration system. The radiometric calibration is better than $\pm 0.5 \mathrm{~dB}$, and the channel imbalance is less than $\pm 0.5 \mathrm{~dB}$ in amplitude and $\pm 5^{\circ}$ in phase [12]. The cross-polarization contamination is generally suppressed by more than $30 \mathrm{~dB}$. The stability of the system is very important in the change detection scheme set up in this paper.

A large number of acquisitions with both the C-and L-band polarimetric SAR from 1994 to 1999 exist for the agricultural test site. To illustrate the change detection capability of the derived test statistic, L-band data from April 17, 1998 and May 


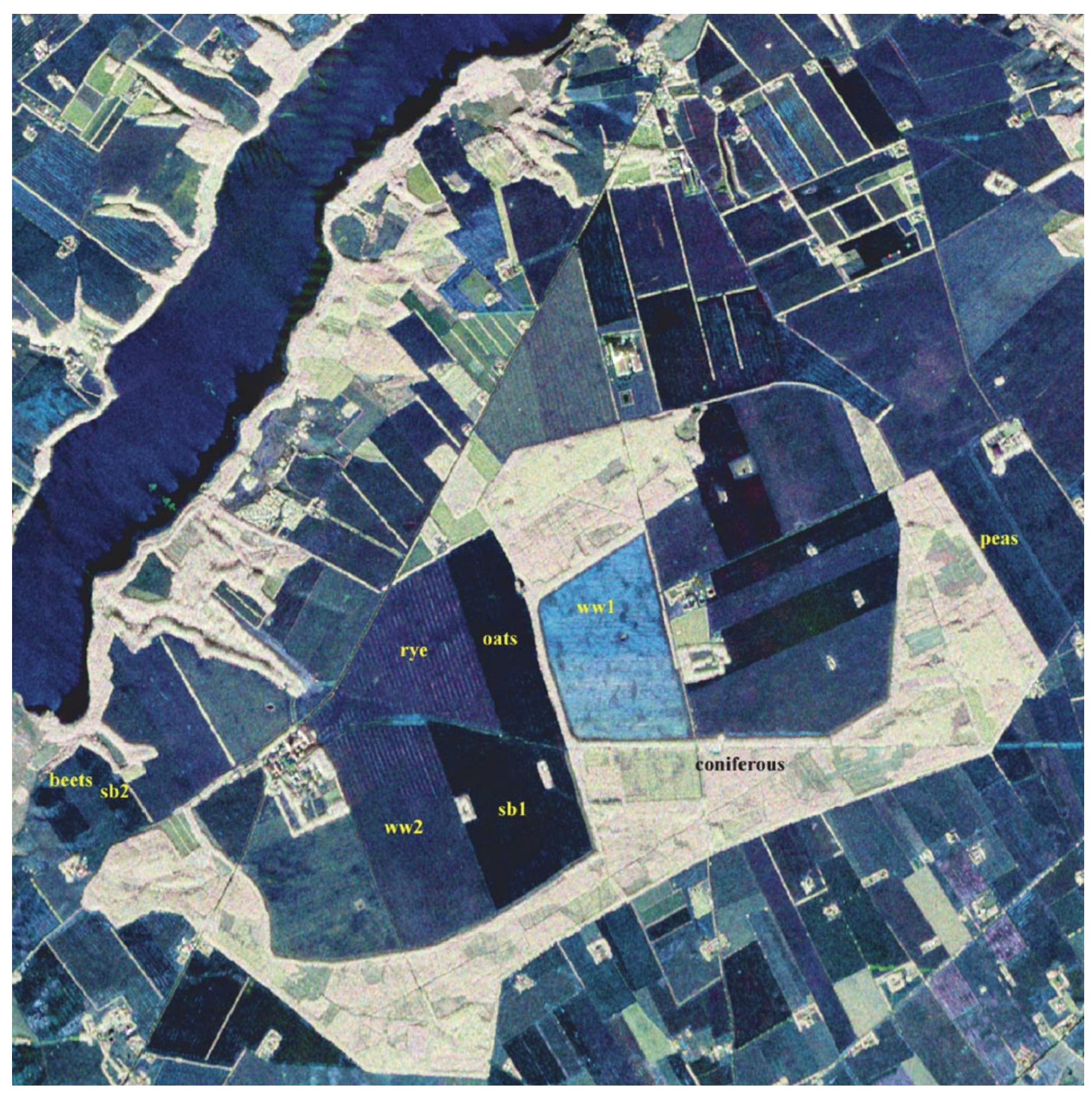

Fig. 2. L-band EMISAR image of the test area acquired on April 17, 1998, $5210 \mathrm{~m} \times 5120 \mathrm{~m}$.

20, 1998 have been used. The two EMISAR images are shown in Figs. 2 and 3, as color composites of the HH (green), HV (actually the complex addition of $\mathrm{HV}$ and $\mathrm{VH}$, red), and VV (blue) channels. The $\mathrm{HH}$ and VV channels are stretched linearly between $-30 \mathrm{~dB}$ and $0 \mathrm{~dB}$. The $\mathrm{HV}$ channel is stretched linearly between $-36 \mathrm{~dB}$ and $-6 \mathrm{~dB}$. The area is relatively flat, and corrections of the local incidence angle due to terrain slope are not critical in this study, since the acquisition geometry for the two acquisitions are almost identical, and therefore the correction has not been carried out. The geometrical coregistration is, however, very important in a change detection application, where two images are compared on a pixel-by-pixel basis. The polari- metric images were registered to a digital elevation model generated from interferometric airborne data acquired by EMISAR. The registration was carried out by combining a model of the imaging geometry with few ground control points, and the images were registered to one another with a root mean-square accuracy of better than one pixel [13]. In the study, 13-look covariance matrix data with a $5 \mathrm{~m} \times 5 \mathrm{~m}$ pixel spacing are used.

\section{B. Test Site}

The area contains a number of agricultural fields of different sizes with different crops. The lengthy, dark blue feature in the upper left corner of Figs. 2 and 3 is a lake, while the bright 


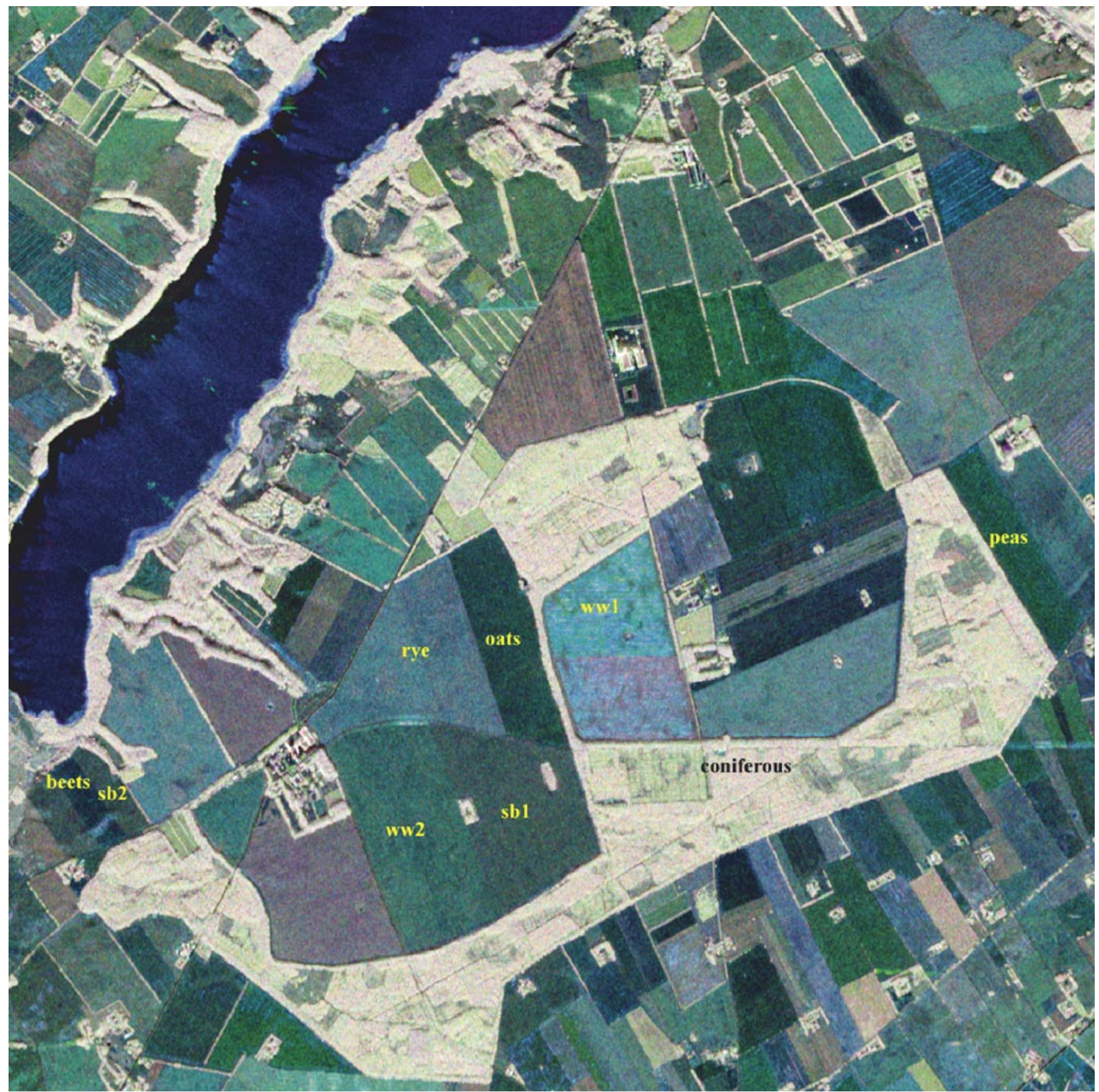

Fig. 3. L-band EMISAR image of the test area acquired on May 20, 1998, $5210 \mathrm{~m} \times 5120 \mathrm{~m}$.

greenish areas seen especially in the lower part of the images are forests (primarily coniferous forests). In the April acquisition, the colors of the agricultural fields are predominantly bluish, due to the larger VV- than HH-backscatter coefficient for the bare fields for the spring crops and the sparsely vegetated fields for the winter crops. For the May acquisition, the spring crops are mainly in the tillering stage, and the winter crops are at the end of the stem elongation stage, in the boot stage, or at the beginning of heading, depending on the crop type.

A number of test areas have been selected for quantitative analysis of the test statistic. These areas are outlined in Figs. 2 and 3, and the development stage and the height of the vegeta- tion are shown in Table I for reference. Five spring crop fields have been used, i.e., one beet field, one pea field, two spring barley fields, and one oats field. All spring crop fields are bare at the April acquisition with the surface being relatively smooth due to sowing or harrowing. At the May acquisition, the beet field is still bare, whereas the other fields have some relatively dense and low vegetation. The three winter crop fields, i.e., two winter wheat fields and one rye field, have low vegetation for the April vegetation and relatively dense and high vegetation for the May acquisition. Finally, a common spruce field, which is virtually unchanged between the two acquisitions, is used in the investigation. 
TABLE I

DEVElopment Stages AND Vegetation HeIghts (IN PARENTHESES)

\begin{tabular}{|c|c|c|}
\hline Crop & April & May \\
\hline Beets & bare (harrowed) & bare \\
\hline Peas & bare (rolled) & middle of side shoot development $(11 \mathrm{~cm})$ \\
\hline Spring barley 1 & bare & end of tillering $(14 \mathrm{~cm})$ \\
\hline Spring barley 2 & bare (sowed) & middle of tillering $(12 \mathrm{~cm})$ \\
\hline Oats & bare & end of tillering $(22 \mathrm{~cm})$ \\
\hline Winter wheat 1 & beginning of stem elongation & end of stem elongation $(44 \mathrm{~cm})$ \\
\hline Winter wheat 2 & end of tillering $(14 \mathrm{~cm})$ & end of stem elongation $(42 \mathrm{~cm})$ \\
\hline Rye & beginning of tillering $(21 \mathrm{~cm})$ & end of boot stage $(51 \mathrm{~cm})$ \\
\hline Coniferous & common spruce & common spruce \\
\hline
\end{tabular}

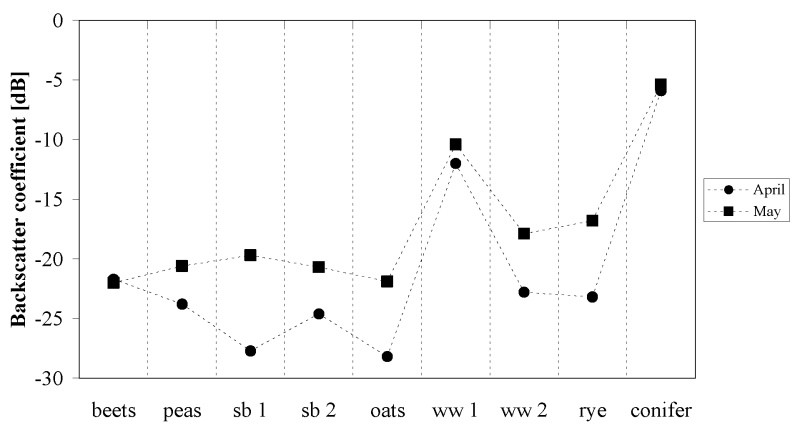

(a)

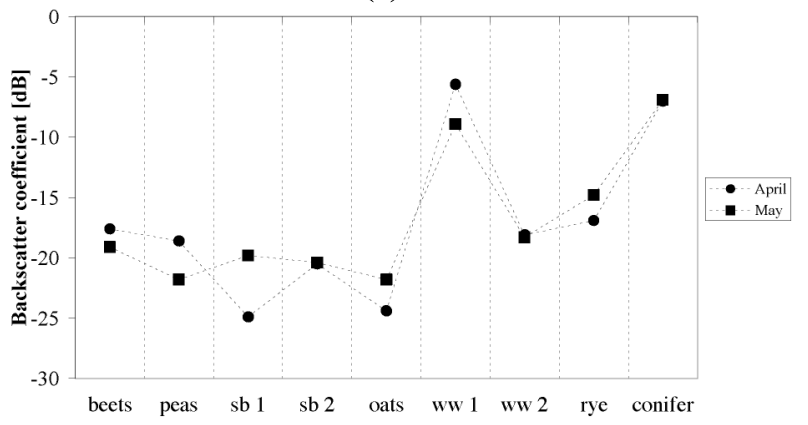

(b)

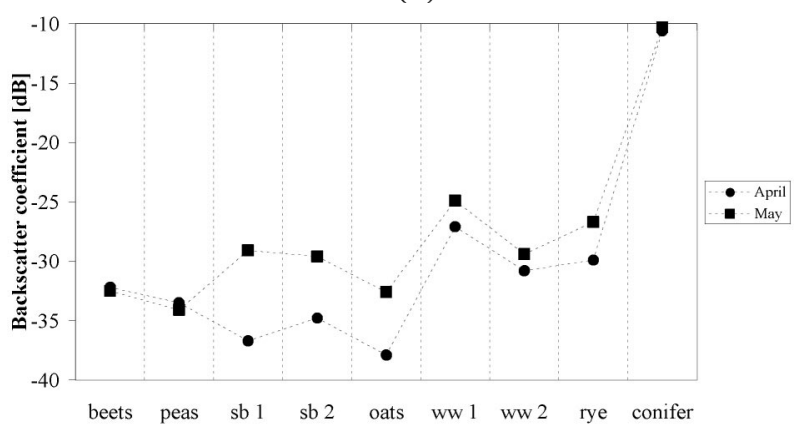

(c)

Fig. 4. (a) $\gamma_{\mathrm{hh}}^{0}$, (b) $\gamma_{\mathrm{vv}}^{0}$, and (c) $\gamma_{\mathrm{hv}}^{0}$ backscatter coefficients for the test areas shown in Figs. 2 and 3 for L-band in April and in May.

\section{RESUlTS AND DISCUSSION}

In Section IV-A, polarimetric parameters for the fields used in the quantitative evaluation will be presented and discussed to provide the background for interpretation of the test statistic results. The results for the test statistic are presented and discussed in Section IV-B.

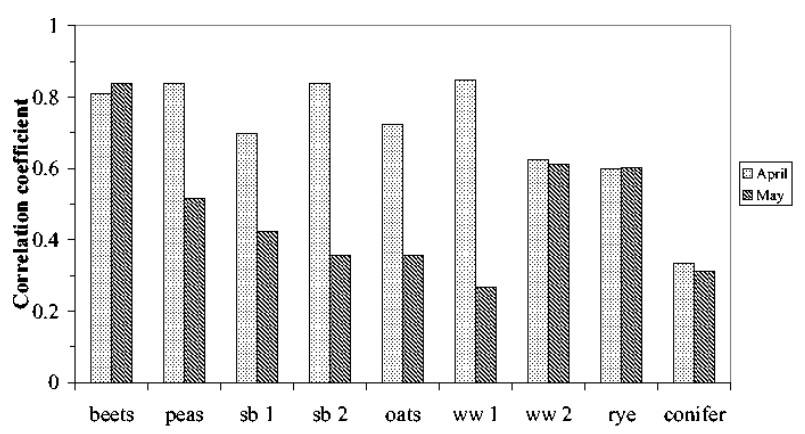

(a)

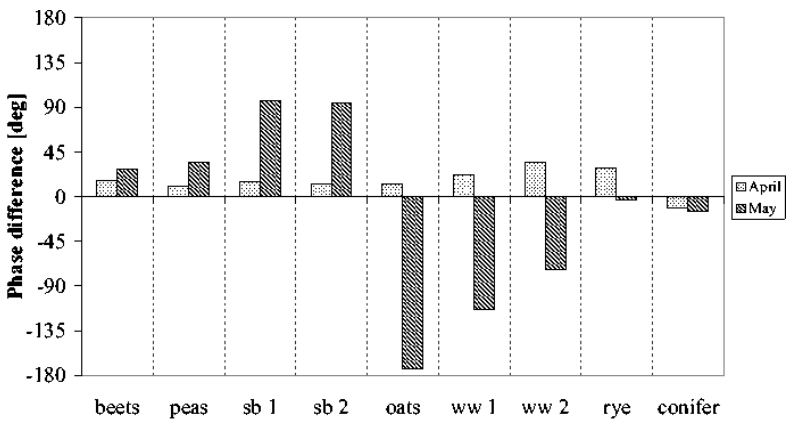

(b)

Fig. 5. (a) Correlation coefficient $\rho_{\mathrm{hhvv}}$ and (b) phase difference $\phi_{\mathrm{hh}-\mathrm{vv}}$ between $\mathrm{HH}$ and VV for the test areas shown in Figs. 2 and 3 for L-band in April and in May.

\section{A. Polarimetric Parameters}

The polarimetric parameters used to describe the selected fields are standard parameters derived from the covariance matrix (1) [2]; the $\gamma^{0}$ backscatter coefficients $\gamma_{\mathrm{hh}}^{0}, \gamma_{\mathrm{hv}}^{0}$, and $\gamma_{\mathrm{vv}}^{0}$ where the $\gamma^{0}$ backscatter coefficient is slightly less dependent on the incidence angle than the $\sigma^{0}$ backscatter coefficient [2]; the correlation coefficient $\rho_{\mathrm{hhvv}}$ and the phase difference $\phi_{\mathrm{hh}-\mathrm{vv}}$ of the $\mathrm{HH}$ and VV components, which contain important information about the scattering mechanisms; and the co- and cross-polarized polarization signatures, which are graphical representations of the polarimetric properties [2], [3]. Fig. 4 shows the $\gamma_{\mathrm{hh}}^{0}, \gamma_{\mathrm{hv}}^{0}$, and $\gamma_{\mathrm{vv}}^{0}$ backscatter coefficients for the various test fields and for both the April and the May acquisitions. Correspondingly, $\rho_{\mathrm{hhvv}}$ and $\phi_{\mathrm{hh}-\mathrm{vv}}$ are shown in Fig. 5, and the polarization responses are shown in Fig. 6.

1) Spring Crops: All spring crops (beets, peas, spring barley 1 and 2, oats) show classical behavior for rough surface scattering for the April acquisition, i.e., high $\rho_{\text {hhvv }}$ [Fig. 5(a)], small $\phi_{\mathrm{hh}-\mathrm{vv}}$ [Fig. 5(b)], low $\gamma_{\mathrm{hv}}^{0}$ backscatter [Fig. 4(c)], larger $\gamma_{\mathrm{vv}}^{0}$ than $\gamma_{\text {hh }}^{0}$ backscatter [Fig. 4(a) and (b)], and textbook examples of surface scattering polarization responses (which are therefore not shown here). The actual backscatter level from the surface is, of course, controlled by the soil moisture and the surface roughness of the individual fields, and we observe rather weak backscatter from the spring barley 1 and the oats fields (Fig. 4) for the April acquisition due to very smooth surfaces.

The beets field also shows rough surface behavior for the May acquisition [Fig. 6(a)]. The pea field shows some volume scattering behavior for the May acquisition, due to the sparse vegetation, i.e., the $\rho_{\text {hhvv }}$ [Fig. 5(a)] has decreased, and the pedestal of the polarization response has increased [Fig. 6(b)]. This effect is 

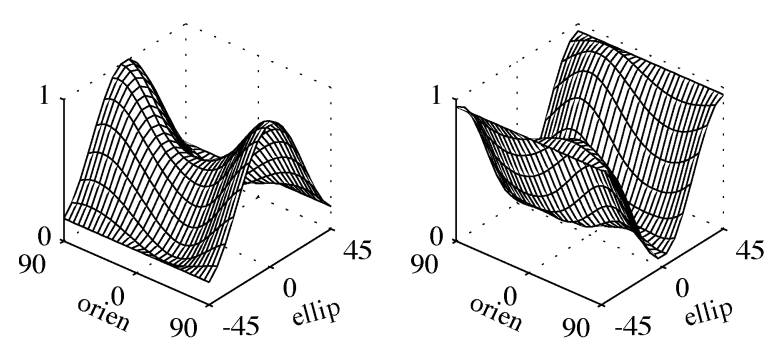

(a) beets, May
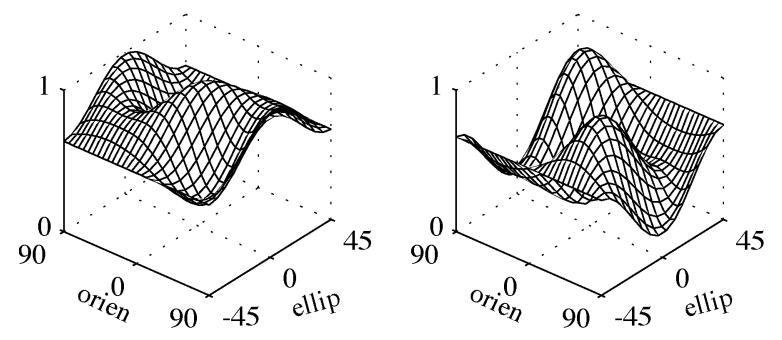

(c) spring barley 1, May
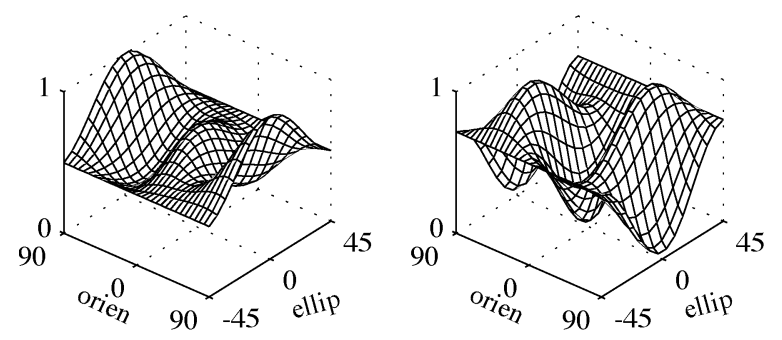

(e) winter wheat 1, May
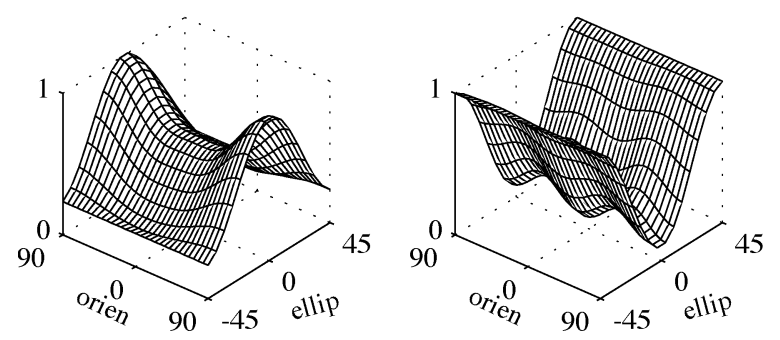

(g) rye, May
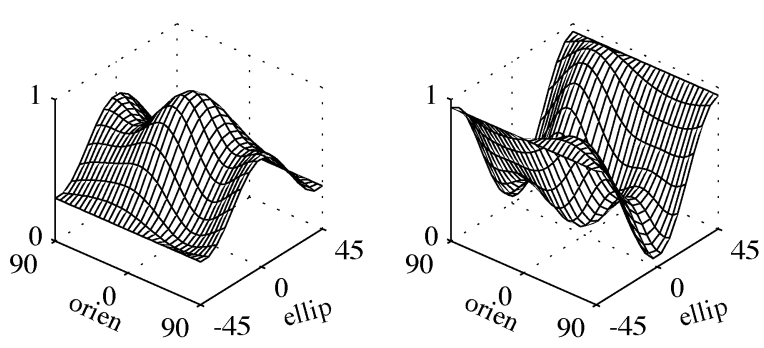

(b) peas, May
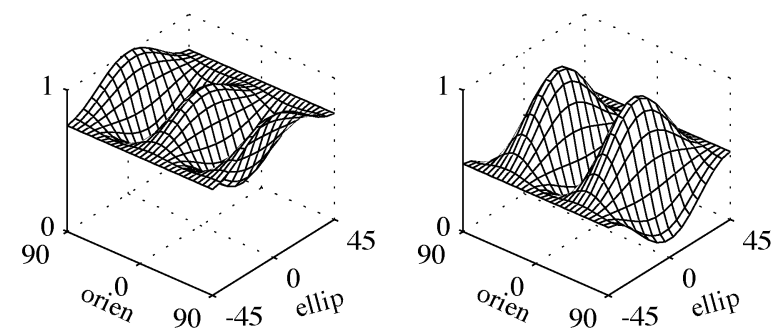

(d) oats, May
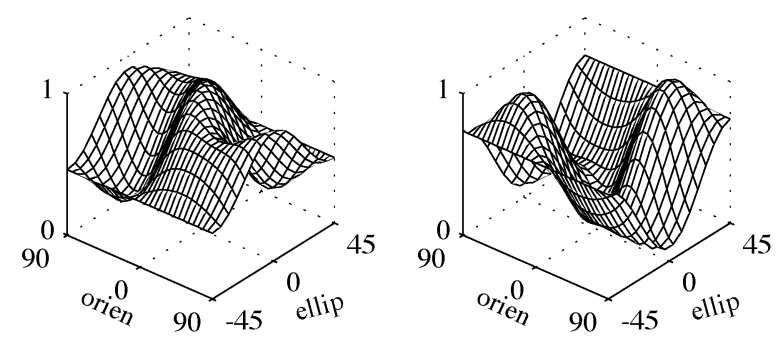

(f) winter wheat 2, May
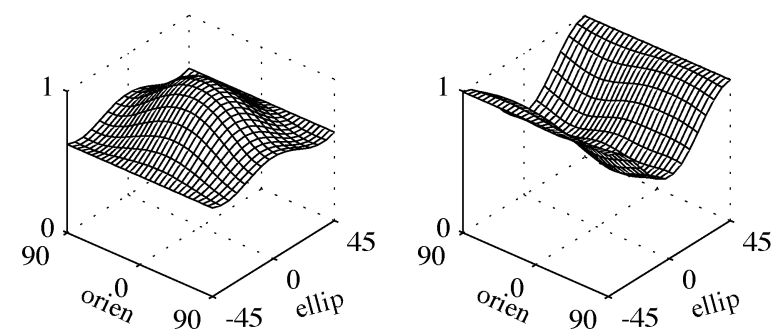

(h) coniferous, May

Fig. 6. Polarization signatures for the test areas shown in Figs. 3 for the L-band acquisition in May. Orientation angle of $0^{\circ}$ corresponds to HH backscatter and the left-hand signature is the copolarized signature, whereas the right-hand signature is the cross-polarized signature.

even more pronounced for the spring barley and the oats fields, due to a more dense vegetation [Figs. 5(a) and 6(c) and (d)]. For the latter fields, a large $\phi_{\mathrm{hh}-\mathrm{vv}}$ is observed too [Fig. 5(b)], and a pronounced double-bounce response is observed, especially for oats [Fig. 6(c) and (d)]. The double-bounce scattering is most likely caused by penetration through the vegetation, scattering from the ground surface, and scattering from the vegetation, or vice versa. This phenomenon has previously been observed early in the growing season for winter crops [2].

2) Winter Crops: The backscatter coefficients from the winter crops are, in general, larger than from the spring crops (Fig. 4), due to the contribution from the volume scattering. The behavior of the winter wheat and the rye fields resembles surface scattering for the April acquisition (Fig. 5), indicating penetration through the vegetation and a large surface scattering component. The cross-polarized backscatter is, however, somewhat larger than for surface scattering, due to the volume scattering contribution [Fig. 4(c)]. The backscattering from the winter wheat 1 field is significantly larger than from the winter wheat 2 field for both acquisitions (Fig. 4). The reason is that the sowing direction for the winter wheat 1 field is exactly perpendicular to the radar look direction. For the May acquisition, the winter wheat fields also show some double-bounce scattering behavior [Figs. 5(b) and 6(e) and (f)]. The rye field shows virtually no change in the polarimetric parameters between the two acquisitions, except for some increase in the $\gamma_{\text {hh }}^{0}$ backscatter (Fig. 4). The coniferous forest area shows pronounced volume scattering behavior for both acquisitions, i.e., 


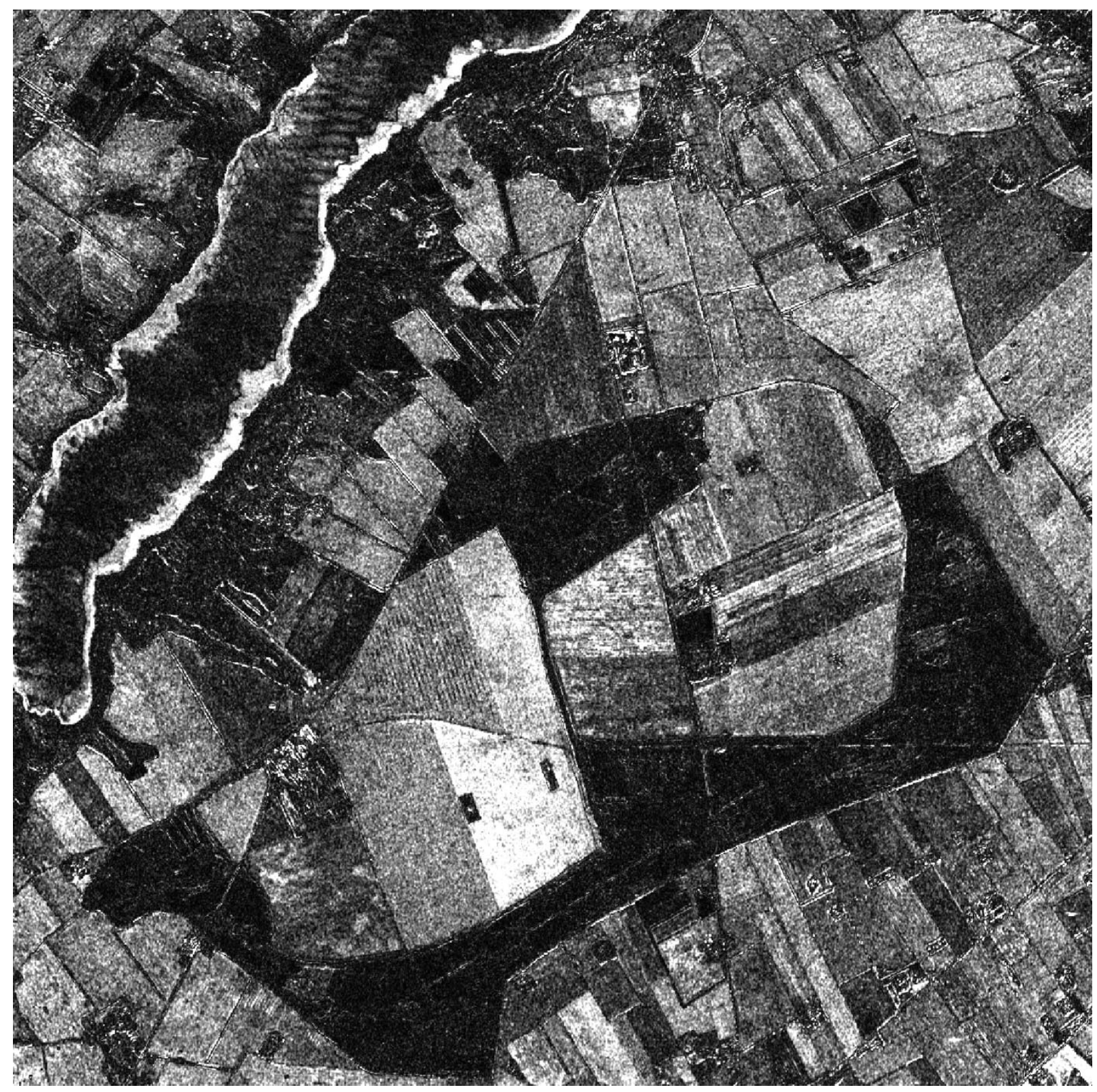

Fig. 7. Logarithm of the test statistic $\ln Q$ (16) for the images shown in Figs. 2 and 3 in the assumed azimuthally symmetric case.

small $\rho_{\text {hhvv }}$ [Fig. 5(a)], small $\phi_{\mathrm{hh}-\mathrm{vv}}$ [Fig. 5(b)], strong $\gamma_{\mathrm{hv}}^{0}$ backscatter [Fig. 4(c)], and large pedestal for the polarization responses [Fig. 6(h)].

\section{B. Test Statistic}

Figs. 7 and 8 show " $-\ln Q$ " (16) for the azimuthally symmetric case and the diagonal-element-only case, respectively, for the two images shown in Figs. 2 and 3. The test statistic is inverted to show areas with large change as bright areas and areas with small change as dark areas. Consequently, when "large values of the test statistic" is mentioned below, it means large values of " $-\ln Q$ " and vice versa for small values. We observe that especially the forest areas appear very dark, indicating virtually no change between the two acquisitions. For the agricultural fields, the results range from dark (no change) to bright (large change) areas depending on the crop type. Fig. 9(a) shows the average " $-\ln Q$ " for the test areas outlined in the previous sections in the following four different cases:

1) using only the VV channel;

2) using only the three diagonal elements of the covariance matrix;

3) using the covariance matrix but assuming azimuthal symmetry;

4) using the full covariance matrix.

Furthermore, Fig. 9(b) shows the average probability of finding a larger value of " $-2 \rho \ln Q$ " (derived from (19) and Theorem 6 in the Appendix) for the four cases mentioned above. Fig. 9(b) also indicates the $5 \%$ and the $1 \%$ significance levels, and the regions with probabilities lower than these levels are the regions where we will typically reject the hypothesis of equal covariance matrices (or VV channels) at the two points in time, i.e., these are regions with major change between the two data acquisitions. 


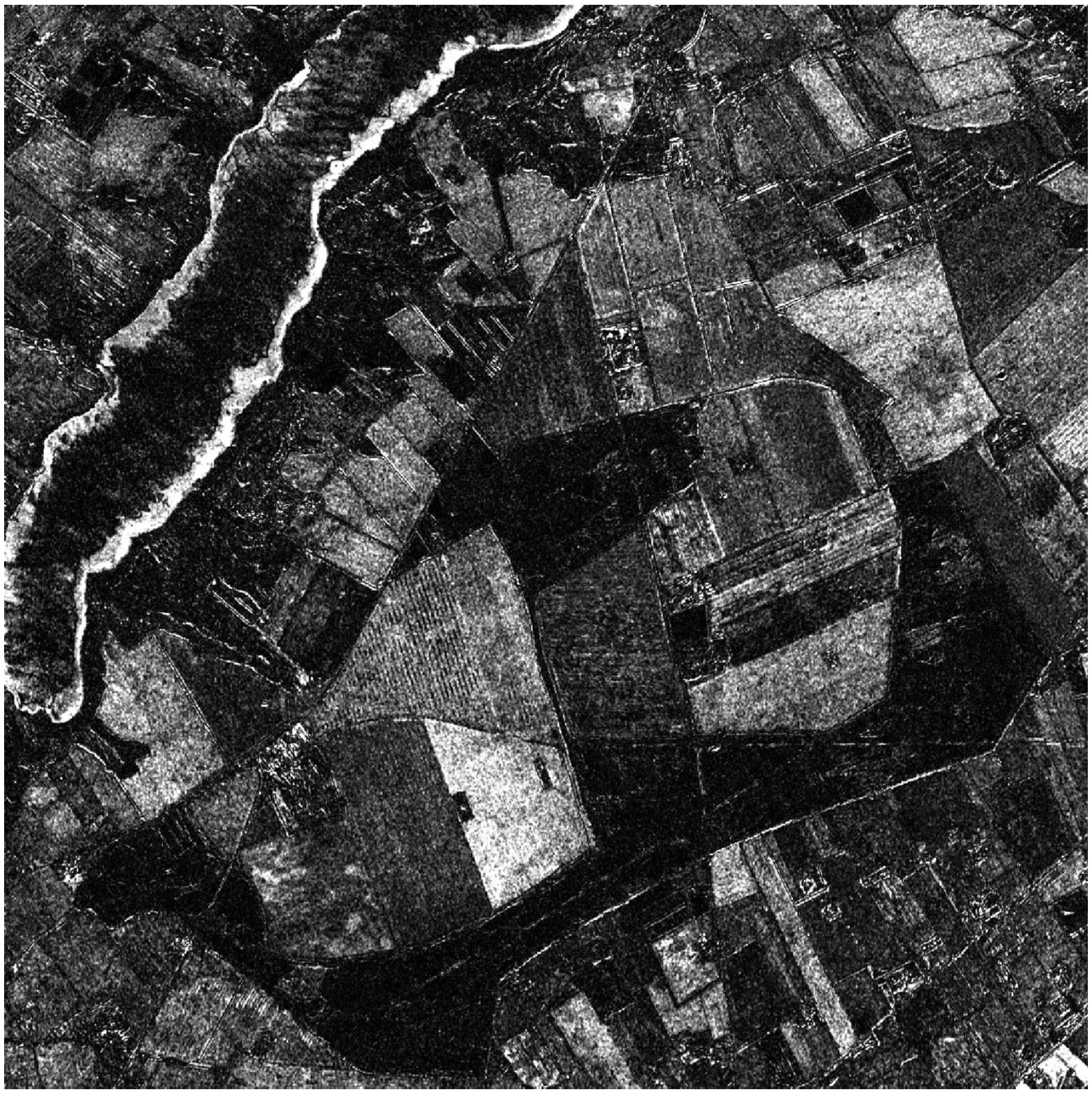

Fig. 8. Logarithm of the test statistic $\ln Q$ (16) for the test areas shown in Figs. 2 and 3 in the diagonal case.

Figs. 10 and 11 show in white for the images in Figs. 2 and 3 , where the hypothesis of equal covariance matrix has been rejected at a $1 \%$ significance level for the azimuthally symmetric case and the diagonal case, respectively. Clearly, we observe detection of changes in the azimuthally symmetric case that have not been detected in the diagonal case, as well as improved detection in the azimuthally symmetric case of changes that already to some extent have been detected in the diagonal case.

In general, the test statistic for the full covariance matrix is only slightly larger than that for the assumed azimuthally symmetric case [Fig. 9(a)]. We may conclude that the additional information added by the co- and cross-elements of the covariance matrix is small. Also, the change detection potential of the single VV channel is seen to be much less than for the other three cases. Therefore, the discussion below will concentrate on comparing the results for two cases: the diagonal case, where only the three diagonal backscatter coefficient elements of the covariance matrix are used, and the polarimetric case, where azimuthal symmetry is assumed (i.e., all the co- and cross-polarization elements are zero).

1) Similar Polarimetric Parameters: The two regions with virtually no change between the acquisitions but with different dominating scattering mechanisms, i.e., beets (surface scattering) and coniferous forest (volume scattering), show both large values of the test statistic and no significant difference between the diagonal and the polarimetric case. It is not possible to reject the hypothesis of equal covariance matrices at a 5\% significance level for any of the regions [Fig. 9(b)]. The rye field also has very similar polarimetric parameters for the two acquisitions, except for $\gamma_{\mathrm{hh}}^{0}$, as mentioned above, and the test statistic for the diagonal and the polarimetric cases are relatively close [Figs. 7-9(a)]. The hypothesis of equal covariance matrices is rejected at a 5\% significance level for both cases. Consequently, in these cases with relatively similar 


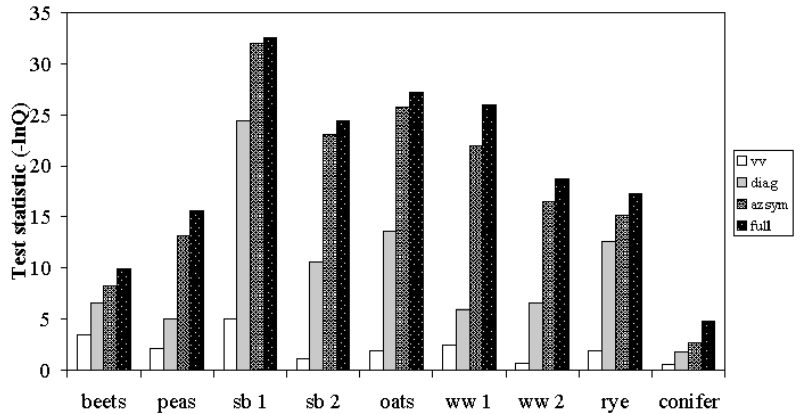

(a)

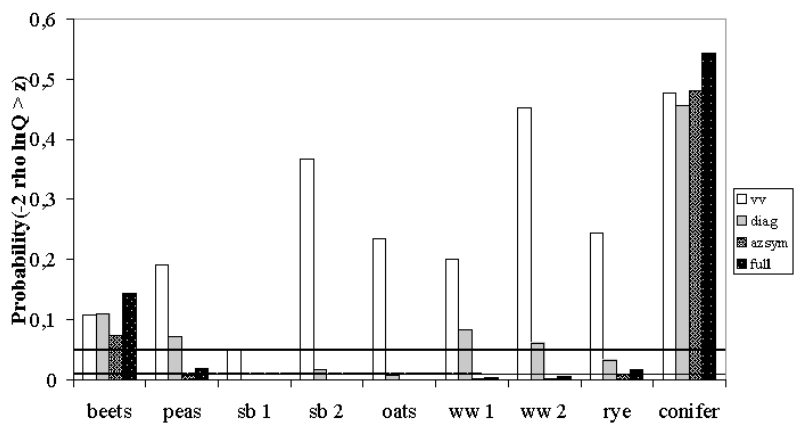

(b)

Fig. 9. (a) Average " $-\ln Q$ " for the test areas shown in Figs. 2 and 3 in four different cases: 1) using only the VV channel, 2) using only the three diagonal elements of the covariance matrix, 3) using the covariance matrix but assuming azimuthal symmetry, and 4) using the full covariance matrix. (b) Average probability of finding a larger value of " $-2 \rho \ln Q$ " (derived from (19) and Theorem 6 in the Appendix) for the same four cases.

polarimetric parameters, the new test statistic for polarimetric data performs equally well as the nonpolarimetric test statistic.

2) Similar Backscatter Coefficients, Large Difference for $\rho_{\mathrm{hhvv}}$ and/or $\phi_{\mathrm{hh}-\mathrm{vv}}$ : Three fields have very similar backscatter coefficients for the two acquisitions, whereas a large difference between $\rho_{\mathrm{hhvv}}$ and/or $\phi_{\mathrm{hh}-\mathrm{vv}}$ exists between the two acquisitions, i.e., the pea field (where $\rho_{\mathrm{hhvv}}$ decreases between the two acquisitions, due to the sparse vegetation cover at the May acquisition), the winter wheat 2 field (where $\phi_{\mathrm{hh}-\mathrm{vv}}$ changes significantly between the two acquisitions), and the winter wheat 1 field (where both $\rho_{\mathrm{hhvv}}$ and $\phi_{\mathrm{hh}-\mathrm{vv}}$ change significantly). A significantly larger test statistic is observed in the polarimetric case than in the diagonal case [Figs. 7-9(a)] for all three fields. Also, it is not possible to reject the hypothesis of equal covariance matrices at a $5 \%$ significance level for any of the three fields in the diagonal case [Fig. 9(b)]. On the other hand, the hypothesis is rejected in the polarimetric case at a $1 \%$ significance level for all three fields [Fig. 9(b)]. Thus, the results clearly show that the new polarimetric test statistic is very sensitive to differences in the full polarimetric information contained in the covariance matrix.

3) Large Difference for All Polarimetric Parameters: Finally, three regions show significant changes in all polarimetric parameters, i.e., the backscatter coefficients, the $\rho_{\mathrm{hhvv}}$, and the $\phi_{\mathrm{hh}-\mathrm{vv}}$, i.e., the two spring barley fields and the oats field, which have a smooth bare surface at the April acquisition and a relatively dense vegetation cover at the May acquisition. For the spring barley 2 and the oats fields, we see a medium test statistic in the diagonal case, whereas a much larger test statistic is observed for the polarimetric case [Figs. 7-9(a)]. The spring barley 1 field has a very large change in the backscatter coefficients, due to the relatively smooth surface at the April acquisition (Fig. 4), and we observe a very large test statistic for both the diagonal and the polarimetric cases [Figs. 7-9(a)]. The two spring barley fields have almost the same $\rho_{\mathrm{hhvv}}$ and $\phi_{\mathrm{hh}-\mathrm{vv}}$ (Fig. 5), whereas the change in the backscatter coefficients is largest for the spring barley 1 field, as mentioned above. This difference is clearly important for both test statistics, where the test statistic for both the diagonal and the polarimetric case is much larger for the spring barley 1 field than for the spring barley 2 field. The hypothesis of equal covariance matrices is rejected for all three fields at the $5 \%$ significance level in both the diagonal and the polarimetric case. This is also the case at the $1 \%$ significance level, except for the spring barley 2 field in the diagonal case. Consequently, even when large changes in the backscatter coefficients ensure detection with a nonpolarimetric method, the addition of polarimetric information improves the detection of changes with the new polarimetric test statistic.

\section{CONCLUSIONS}

In this paper, a test statistic for equality of two complex Wishart distributed covariance matrices and an associated asymptotic probability measure for obtaining a smaller value of the test statistic have been derived. The test statistic provides a unique opportunity to develop optimal algorithms for change detection, edge detection, line detection, segmentation, etc., for polarimetric SAR images. Such algorithms have previously been based on results of applying algorithms to the single channels and subsequently combining these results using some kind of fusion operator.

As a demonstration of the potential of the new test statistic, the derived measures have been applied to change detection in fully polarimetric SAR data for a test area with primarily agricultural fields and forest stands where two images acquired with approximately one-month interval have been used. In the case with areas with only small change in the polarimetric parameters between the two acquisitions, the new test statistic for polarimetric data performs equally well as the nonpolarimetric test statistic. When the backscatter coefficients are virtually unchanged, but either the phase and/or the correlation coefficient between the $\mathrm{HH}$ and VV polarizations have changed, the results clearly show that the new polarimetric test statistic is much more sensitive to the differences than test statistics based only on the backscatter coefficients. Also, in the case where all parameters in the covariance matrix have changed between the two polarizations, the new test statistic shows improved change detection capability. Consequently, the results show clearly that the new test statistic offers improved change detection capability for fully polarimetric SAR data.

\section{APPENDIX}

\section{ANALYSIS OF COMPLEX WISHART MATRICES}

In change detection and edge detection in polarimetric SAR data, it is useful to be able to compare two complex Wishart distributed matrices. 


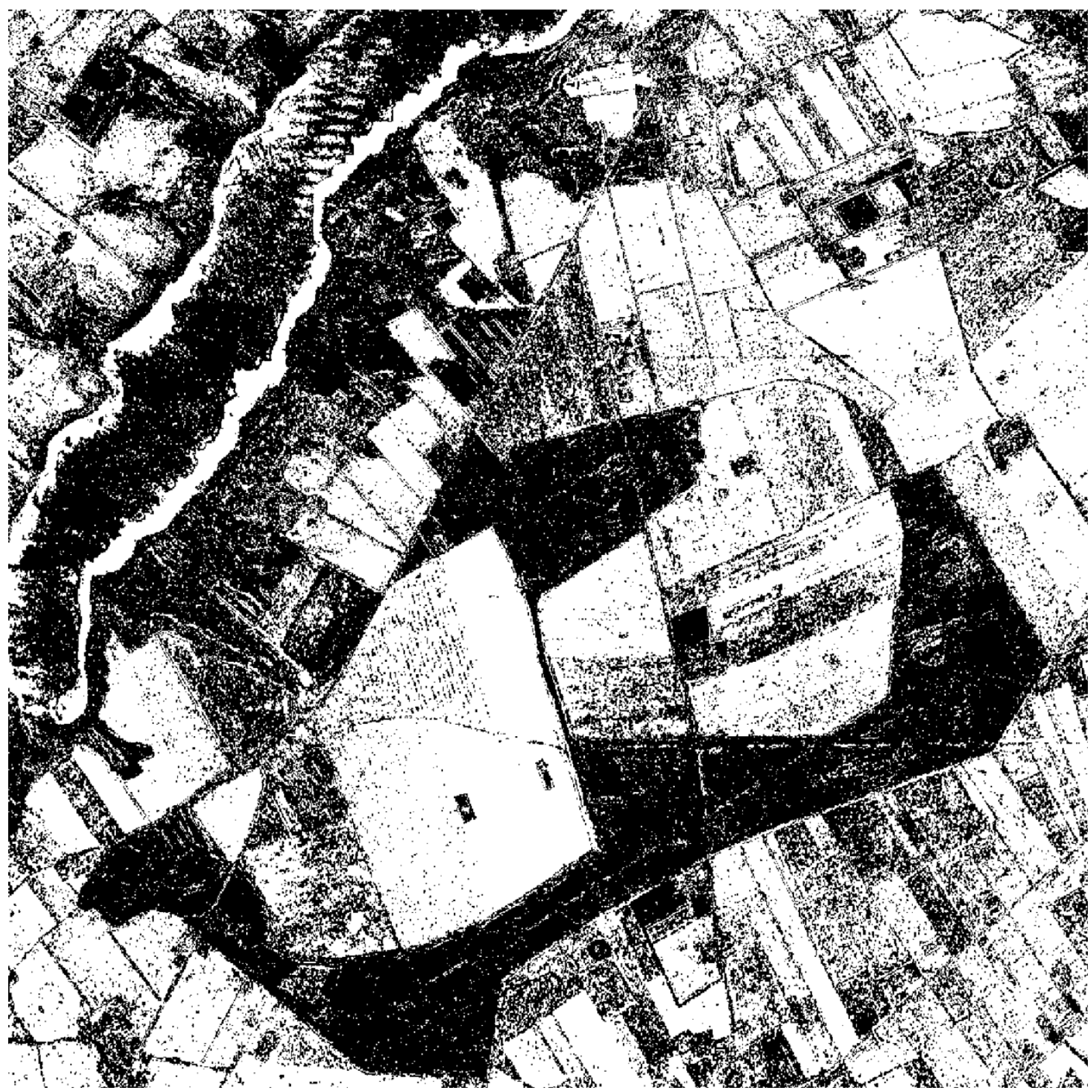

Fig. 10. Rejection of hypothesis of equal covariance matrices at $1 \%$ level for the assumed azimuthally symmetric case (white: rejection, black: acceptance).

Most of the standard literature in multivariate analysis only contains references to the real case (e.g., see [16]). This does not mean, however, that results for the complex case do not exist. In [4], the relevant class of complex distributions is introduced, and [17] completed much of the work, either giving results or (indirectly) pointing out how results may be obtained. It is, however, not straightforward to deduce the relevant formulas from their work.

In [18], many of the necessary formulas are deduced in an elegant way using the fact that the problem is invariant under a group of linear transformations. The notation chosen is not straightforward however. Some of their results appear in [19], an unpublished thesis in Danish, including results on comparing covariance matrices. In [20], the theory for linear and graphical models in multivariate complex normal models is covered.

Since the formulas for the distribution of the likelihood ratio do not seem to be available and since no authors seem to have treated the so-called block diagonal case, we have chosen to give a rather thorough description of the necessary results.

We start with a short introduction to the complex normal and the complex Wishart distributions. We then compare two gamma distributions, which is the one-dimensional test often usen in the radar community. Then we give a straightforward (brute force) derivation of the likelihood-ratio criterion for testing the equality in the complex case. Then, we describe the so-called block diagonal case, which among other things covers the case known in the radar community as the azimuthal symmetric case and the total independence case.

After quoting results from [21] on asymptotic distributions, we establish the necessary results on moments by brute force integration. By straightforward but rather cumbersome calculations, the results in [21] yield the desired results. Alternatively, one may use results in [20] and derive expressions involving the product of beta distributed random variables. 


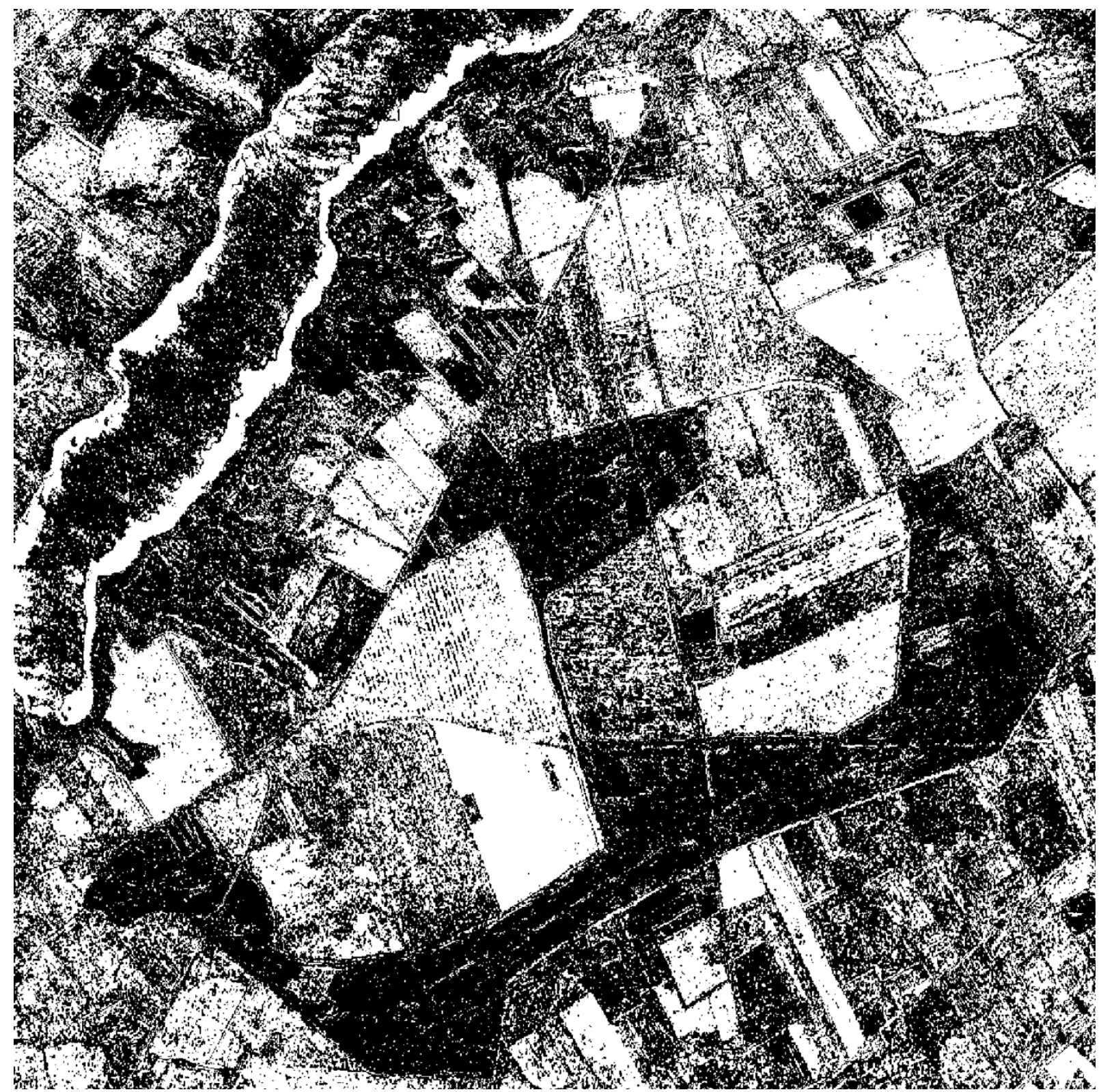

Fig. 11. Rejection of hypothesis of equal covariance matrices at $1 \%$ level for the diagonal case (white: rejection, black: acceptance).

\section{A. Complex Normal Distribution}

Following [4], we say that a $p$-dimensional random complex vector $\boldsymbol{Z}$ follows a complex multivariate normal distribution with mean $\mathbf{0}$ and dispersion matrix $\boldsymbol{\Sigma}$, i.e.,

$$
\boldsymbol{Z}=\left[Z_{1} \cdots Z_{p}\right]^{T} \in N_{C}(\mathbf{0}, \mathbf{\Sigma})
$$

if the frequency function is

$$
\begin{aligned}
f(z) & =\frac{1}{\pi^{p}|\boldsymbol{\Sigma}|} \exp \left\{-z^{* T} \boldsymbol{\Sigma}^{-1} z\right\} \\
& =\frac{1}{\pi^{p}|\boldsymbol{\Sigma}|} \exp \left\{-\operatorname{tr}\left[\boldsymbol{\Sigma}^{-1} z z^{* T}\right]\right\} .
\end{aligned}
$$

$\boldsymbol{\Sigma}$ is Hermitian positive definite and of the form

$$
\left[\begin{array}{ccc}
\sigma_{1}^{2} & \left(\alpha_{12}+i \beta_{12}\right) \sigma_{1} \sigma_{2} & \cdots \\
\left(\alpha_{12}-i \beta_{12}\right) \sigma_{1} \sigma_{2} & \sigma_{2}^{2} & \cdots \\
\left(\alpha_{13}-i \beta_{13}\right) \sigma_{1} \sigma_{3} & \left(\alpha_{23}-i \beta_{23}\right) \sigma_{2} \sigma_{3} & \cdots \\
\vdots & \vdots & \ddots
\end{array}\right] .
$$

In other words, we have for $Z_{i}=X_{i}+i Y_{i}$

$$
\begin{aligned}
E\left\{Z_{j} Z_{j}^{*}\right\} & =E\left\{X_{j}^{2}+Y_{j}^{2}\right\}=\sigma_{j}^{2} \\
E\left\{Z_{j} Z_{k}^{*}\right\} & =\left(\alpha_{j k}+i \beta_{j k}\right) \sigma_{j} \sigma_{k} .
\end{aligned}
$$

\section{B. Complex Wishart Distribution}

We say that a Hermitian positive definite random $p \times p$ matrix $\boldsymbol{X}$ follows a complex Wishart distribution, i.e.,

$$
\boldsymbol{X} \in W_{C}(p, n, \boldsymbol{\Sigma})
$$


if the frequency function is

$$
w(\boldsymbol{x})=\frac{1}{\Gamma_{p}(n)} \frac{1}{|\boldsymbol{\Sigma}|^{n}}|\boldsymbol{x}|^{n-p} \exp \left\{-\operatorname{tr}\left[\boldsymbol{\Sigma}^{-1} \boldsymbol{x}\right]\right\}
$$

where

$$
\Gamma_{p}(n)=\pi^{p(p-1) / 2} \prod_{j=1}^{p} \Gamma(n-j+1)
$$

If confusion concerning $n$ or $\boldsymbol{\Sigma}$ may arise, we write $w(\boldsymbol{x} ; n, \boldsymbol{\Sigma})$ rather than $w(\boldsymbol{x})$. The frequency function is defined for $\boldsymbol{x}$ positive definite, and in evaluating integrals the volume element becomes

$$
d \boldsymbol{x}=\left(d x_{11}, \ldots, d x_{p p}\right) \prod_{k=1}^{p} \prod_{j=1}^{k-1} d x_{j k R} d x_{j k I}
$$

where $R$ and $I$ denote real and imaginary parts. For further description and useful results on Jacobians, etc., see [4] and [17].

It is emphasized that the formulas for the complex normal and Wishart distributions differ from their real counterparts.

Estimation of $\boldsymbol{\Sigma}$ from a normal sample follows in Theorem 1.

Theorem 1: Let $\boldsymbol{Z}_{1}, \ldots, \boldsymbol{Z}_{n}$ be independent, complex normal random variables, i.e.,

$$
Z_{i} \in N_{C}(\mathbf{0}, \boldsymbol{\Sigma})
$$

Then the maximum-likelihood (ML) estimator for $\boldsymbol{\Sigma}$ is

$$
\hat{\Sigma}=\frac{1}{n} \sum_{j=1}^{n} Z_{j} Z_{j}^{* T}
$$

and $n \hat{\Sigma}$ is Wishart distributed

$$
n \hat{\boldsymbol{\Sigma}} \in W_{C}(p, n, \boldsymbol{\Sigma})
$$

Proof: See [4].

From Theorem 1, we easily obtain Theorem 2.

Theorem 2: Let $\boldsymbol{X}$ and $\boldsymbol{Y}$ be independent Wishart distributed matrices

$$
\begin{aligned}
& \boldsymbol{X} \in W_{C}(p, n, \boldsymbol{\Sigma}) \\
& \boldsymbol{Y} \in W_{C}(p, m, \boldsymbol{\Sigma})
\end{aligned}
$$

Then the sum will again be Wishart distributed, i.e.,

$$
\boldsymbol{S}=\boldsymbol{X}+\boldsymbol{Y} \in W_{C}(p, n+m, \boldsymbol{\Sigma})
$$

Proof: Straightforward.

\section{Test on Equality of Two Gamma Parameters}

Let the independent random variables $X$ and $Y$ (which are real scalars) be gamma distributed $G\left(n, \beta_{x}\right)$ and $G\left(m, \beta_{y}\right)$. The frequency function for $X$ is

$$
f\left(x ; \beta_{x}\right)=\frac{1}{\Gamma(n)} \frac{1}{\beta_{x}^{n}} x^{n-1} \exp \left\{\frac{-x}{\beta_{x}}\right\}, \quad x>0
$$

and similarly for $Y$. The likelihood function for the parameters $\left(\beta_{x}, \beta_{y}\right)$ thus becomes

$$
\begin{aligned}
L_{x}\left(\beta_{x}\right) L_{y}\left(\beta_{y}\right)= & \frac{1}{\Gamma(n)} \frac{1}{\Gamma(m)} \frac{1}{\beta_{x}^{n}} \frac{1}{\beta_{y}^{m}} \\
& \cdot X^{n-1} Y^{m-1} \exp \left\{-\frac{X}{\beta_{x}}-\frac{Y}{\beta_{y}}\right\}
\end{aligned}
$$

and under the hypothesis $\beta_{x}=\beta_{y}=\beta$, we obtain

$$
\begin{aligned}
L(\beta)=\frac{1}{\Gamma(n)} \frac{1}{\Gamma(m)} & \frac{1}{\beta^{n+m}} \\
& \cdot X^{n-1} Y^{m-1} \exp \left\{\frac{-(X+Y)}{\beta}\right\} .
\end{aligned}
$$

Taking the derivatives of the log likelihoods and setting them equal to zero, we obtain the ML estimates

$$
\begin{aligned}
\hat{\beta_{x}} & =\frac{X}{n} \\
\hat{\beta}_{y} & =\frac{Y}{m} \\
\hat{\beta} & =\frac{(X+Y)}{(n+m)} .
\end{aligned}
$$

Therefore the likelihood-ratio test statistic becomes

$$
Q=\frac{L(\hat{\beta})}{L_{x}\left(\hat{\beta_{x}}\right) L_{y}\left(\hat{\beta}_{y}\right)}=\frac{(n+m)^{n+m}}{n^{n} m^{m}} \frac{X^{n} Y^{m}}{(X+Y)^{n+m}}
$$

The critical region is given by $Q \leq q$ or

$$
\frac{X^{n} Y^{m}}{(X+Y)^{n+m}} \leq c .
$$

Straightforward calculations show that this critical region is of the form

$$
\frac{Y}{X} \leq c_{1} \text { or } \frac{Y}{X} \geq c_{2} .
$$

Since $Y / X$ under the null hypothesis is distributed like Fisher's $F$, i.e.,

$$
\frac{m}{n} \frac{Y}{X} \in F(2 m, 2 n)
$$

$c_{1}$ and $c_{2}$ may be determined by means of quantiles in the $F$-distribution.

\section{Test on Equality of Two Complex Wishart Matrices}

We consider independent Wishart distributed matrices

$$
\begin{aligned}
& \boldsymbol{X} \in W_{C}\left(p, n, \boldsymbol{\Sigma}_{x}\right) \\
& \boldsymbol{Y} \in W_{C}\left(p, m, \boldsymbol{\Sigma}_{y}\right)
\end{aligned}
$$

and wish to test the hypothesis

$$
H_{0}: \boldsymbol{\Sigma}_{x}=\boldsymbol{\Sigma}_{y}=\boldsymbol{\Sigma} \text { against } H_{1}: \boldsymbol{\Sigma}_{x} \neq \boldsymbol{\Sigma}_{y}
$$


We have the likelihood functions

$$
\begin{aligned}
L_{x}\left(\boldsymbol{\Sigma}_{x}\right) L_{y}\left(\boldsymbol{\Sigma}_{y}\right)= & \frac{1}{\Gamma_{p}(n) \Gamma_{p}(m)} \\
& \cdot\left|\boldsymbol{\Sigma}_{x}\right|^{-n}\left|\boldsymbol{\Sigma}_{y}\right|^{-m}|\boldsymbol{X}|^{n-p}|\boldsymbol{Y}|^{m-p} \\
& \cdot \exp \left\{-\operatorname{tr}\left[\boldsymbol{\Sigma}_{x}^{-1} \boldsymbol{X}+\boldsymbol{\Sigma}_{y}^{-1} \boldsymbol{Y}\right]\right\} \\
L(\boldsymbol{\Sigma})= & \frac{1}{\Gamma_{p}(n) \Gamma_{p}(m)} \\
& \cdot|\boldsymbol{\Sigma}|^{-(n+m)}|\boldsymbol{X}|^{n-p}|\boldsymbol{Y}|^{m-p} \\
& \cdot \exp \left\{-\operatorname{tr}\left[\boldsymbol{\Sigma}^{-1}(\boldsymbol{X}+\boldsymbol{Y}]\right\}\right.
\end{aligned}
$$

The ML estimates are

$$
\begin{aligned}
\hat{\boldsymbol{\Sigma}}_{x} & =\frac{1}{n} \boldsymbol{X} \\
\hat{\boldsymbol{\Sigma}}_{y} & =\frac{1}{m} \boldsymbol{Y} \\
\hat{\boldsymbol{\Sigma}} & =\frac{1}{n+m}(\boldsymbol{X}+\boldsymbol{Y}) .
\end{aligned}
$$

Therefore, the likelihood-ratio test statistic becomes

$$
Q=\frac{L(\hat{\boldsymbol{\Sigma}})}{L_{x}\left(\hat{\boldsymbol{\Sigma}}_{x}\right) L_{y}\left(\hat{\boldsymbol{\Sigma}}_{y}\right)}=\frac{(n+m)^{p(n+m)}}{n^{p n} m^{p m}} \frac{|\boldsymbol{X}|^{n}|\boldsymbol{Y}|^{m}}{|\boldsymbol{X}+\boldsymbol{Y}|^{n+m}} .
$$

Thus, we get the critical region

$$
C=\left\{\boldsymbol{X}, \boldsymbol{Y} \mid \frac{(n+m)^{p(n+m)}}{n^{p n} m^{p m}} \frac{|\boldsymbol{X}|^{n}|\boldsymbol{Y}|^{m}}{|\boldsymbol{X}+\boldsymbol{Y}|^{n+m}} \leq q\right\} .
$$

\section{E. Tests in the Block Diagonal Case}

In some applications (e.g., remote sensing), there are several independent Wishart matrices in each observation. They will often be arranged in a block diagonal structure like

$$
\boldsymbol{X}=\left[\begin{array}{cc}
\boldsymbol{X}_{1} & \mathbf{0}_{p_{1} p_{2}} \\
\mathbf{0}_{p_{2} p_{1}} & \boldsymbol{X}_{2}
\end{array}\right]=\left[\begin{array}{cc}
\boldsymbol{X}_{1} & \mathbf{0} \\
\mathbf{0} & \boldsymbol{X}_{2}
\end{array}\right]
$$

where $\boldsymbol{X}_{i} \in W_{C}\left(p_{i}, n, \boldsymbol{\Sigma}_{x i}\right)$. This covers the so-called azimuthal symmetric case and the case with independence of coand cross-polarized signals. If we define

$$
\boldsymbol{\Sigma}_{x}=\left[\begin{array}{cc}
\boldsymbol{\Sigma}_{x 1} & \mathbf{0} \\
\mathbf{0} & \boldsymbol{\Sigma}_{x 2}
\end{array}\right]
$$

it is important to note that $\boldsymbol{X}$ is not Wishart distributed $W_{C}\left(p_{1}+\right.$ $\left.p_{2}, n, \boldsymbol{\Sigma}_{x}\right)$.

We now consider similar partitionings of $\boldsymbol{X}$ and $\boldsymbol{Y}$, i.e., we have independent random matrices

$$
\begin{aligned}
& \boldsymbol{X}_{i} \in W_{C}\left(p_{i}, n, \boldsymbol{\Sigma}_{x i}\right) \\
& \boldsymbol{Y}_{i} \in W_{C}\left(p_{i}, m, \boldsymbol{\Sigma}_{y i}\right)
\end{aligned}
$$

for $i=1, \ldots, I$. We have $p=p_{1}+\cdots+p_{I}$ and set $N=n+m$. We want to test the hypothesis

$$
\boldsymbol{\Sigma}_{x i}=\boldsymbol{\Sigma}_{y i}=\boldsymbol{\Sigma}_{i}, \quad i=1, \ldots, I
$$

against all alternatives. The likelihood-ratio criterion becomes the product of the criteria, i.e.,

$$
Q=\prod_{i=1}^{I} Q_{i}=\prod_{i=1}^{I} \frac{N^{p_{i} N}}{n^{p_{i} n^{2}} m^{p_{i} m}} \frac{\left|\boldsymbol{X}_{i}\right|^{n}\left|\boldsymbol{Y}_{i}\right|^{m}}{\left|\boldsymbol{X}_{i}+\boldsymbol{Y}_{i}\right|^{n+m}}
$$

Since the determinant of a block diagonal matrix is the product of the determinants of the diagonal elements, we obtain

$$
Q=\frac{N^{p N}}{n^{p n} m^{p m}} \frac{|\boldsymbol{X}|^{n}|\boldsymbol{Y}|^{m}}{|\boldsymbol{X}+\boldsymbol{Y}|^{n+m}}
$$

i.e., the same result as in the general case [see (61)]. Note, however, that the distribution has changed, since $\boldsymbol{X}$ and $\boldsymbol{Y}$ are no longer Wishart distributed.

\section{F. Large Sample Distribution Theory}

In [21] (as quoted in [16]), a general asymptotic expansion of the distribution of a random variable whose moments are certain functions of gamma functions has been developed. We state the main result as a theorem, and we shall use it in determining the (asymptotic) distribution of the likelihood-ratio criterion.

Theorem 3: Let the random variable $W(0 \leq W \leq 1)$ have the $h$ th moment

$$
E\left\{W^{h}\right\}=K\left(\frac{\prod_{j=1}^{b} y_{j}^{y_{j}}}{\prod_{k=1}^{a} x_{k}^{x_{k}}}\right)^{h} \frac{\prod_{k=1}^{a} \Gamma\left(x_{k}(1+h)+\xi_{k}\right)}{\prod_{j=1}^{b} \Gamma\left(y_{j}(1+h)+\eta_{j}\right)}
$$

where $K$ is a constant so that $E\left\{W^{0}\right\}=1$ and $\sum_{k=1}^{a} x_{k}=$ $\sum_{j=1}^{b} y_{j}$. For an arbitrary $\rho$ we set

$$
\begin{aligned}
\beta_{k} & =(1-\rho) x_{k} \\
\epsilon_{j} & =(1-\rho) y_{j} .
\end{aligned}
$$

The first three Bernoulli polynomials are denoted

$$
\begin{aligned}
& B_{1}(h)=h-\frac{1}{2} \\
& B_{2}(h)=h^{2}-h+\frac{1}{6} \\
& B_{3}(h)=h^{3}-\frac{3}{2} h^{2}+\frac{1}{2} h .
\end{aligned}
$$

We define

$$
\begin{array}{r}
f=-2\left[\sum_{k=1}^{a} \xi_{k}-\sum_{j=1}^{b} \eta_{j}-\frac{1}{2}(a-b)\right] \\
\omega_{r}=\frac{(-1)^{r+1}}{r(r+1)}\left\{\sum_{k=1}^{a} \frac{B_{r+1}\left(\beta_{k}+\xi_{k}\right)}{\left(\rho x_{k}\right)^{r}}\right. \\
\left.-\sum_{j=1}^{b} \frac{B_{r+1}\left(\epsilon_{j}+\eta_{j}\right)}{\left(\rho y_{j}\right)^{r}}\right\} .
\end{array}
$$

If we select $\rho$ so that $\omega_{1}=0$, we have

$$
\begin{aligned}
& P\{-2 \rho \ln W \leq z\} \simeq P\left\{\chi^{2}(f) \leq z\right\} \\
& +\omega_{2}\left[P\left\{\chi^{2}(f+4) \leq z\right\}-P\left\{\chi^{2}(f) \leq z\right\}\right] .
\end{aligned}
$$

Proof: See [16]. 
Theorem 4: Let

$$
\begin{aligned}
& \boldsymbol{X} \in W_{C}(p, n, \boldsymbol{\Sigma}) \\
& \boldsymbol{Y} \in W_{C}(p, m, \boldsymbol{\Sigma})
\end{aligned}
$$

be independent complex Wishart distributed matrices. Then for $N=n+m$ and

$$
U=\frac{|X|^{n}|Y|^{m}}{|X+Y|^{N}}
$$

we have

$$
E\left\{U^{h}\right\}=\frac{\Gamma_{p}(n(1+h)) \Gamma_{p}(m(1+h))}{\left.\Gamma_{p}(N(1+h))\right)} \frac{\Gamma_{p}(N)}{\Gamma_{p}(n) \Gamma_{p}(m)} .
$$

Proof: The joint frequency function of $(\boldsymbol{X}, \boldsymbol{Y})$ is $w(\boldsymbol{x} ; n, \boldsymbol{\Sigma}) w(\boldsymbol{y} ; m, \boldsymbol{\Sigma})$. Therefore

$$
E\left\{U^{h}\right\}=\iint u^{h} w(\boldsymbol{x} ; n, \boldsymbol{\Sigma}) w(\boldsymbol{y} ; m, \boldsymbol{\Sigma}) d \boldsymbol{x} d \boldsymbol{y}
$$

and evaluation of this integral gives the desired result.

By means of the two previous theorems, we are now able to state the important result on the (asymptotic) distribution of the likelihood-ratio criterion.

Theorem 5: We consider the likelihood-ratio criterion

$$
Q=\frac{N^{p N}}{n^{p n} m^{p m}} \frac{|\boldsymbol{X}|^{n}|\boldsymbol{Y}|^{m}}{|\boldsymbol{X}+\boldsymbol{Y}|^{N}}
$$

and define

$$
\begin{aligned}
f= & p^{2} \\
\rho= & 1-\frac{2 f-1}{6 \sqrt{f}}\left(\frac{1}{n}+\frac{1}{m}-\frac{1}{N}\right) \\
\omega_{2}= & -\frac{f}{4}\left(1-\frac{1}{\rho}\right)^{2}+\frac{f(f-1)}{24} \\
& \cdot\left(\frac{1}{n^{2}}+\frac{1}{m^{2}}-\frac{1}{N^{2}}\right) \frac{1}{\rho^{2}} .
\end{aligned}
$$

Then

$$
\begin{aligned}
& P\{-2 \rho \ln Q \leq z\} \simeq P\left\{\chi^{2}(f) \leq z\right\} \\
& +\omega_{2}\left[P\left\{\chi^{2}(f+4) \leq z\right\}-P\left\{\chi^{2}(f) \leq z\right\}\right] .
\end{aligned}
$$

Proof: Omitted, straightforward but cumbersome calculations.

We now address the block diagonal case and state the main result in Theorem 6.

Theorem 6: Let the situation be as described in Section A-E, and define for $i=1, \ldots, I$

$$
\begin{gathered}
f_{i}=p_{i}^{2} \\
\rho_{i}=1-\frac{2 f_{i}-1}{6 \sqrt{f_{i}}}\left(\frac{1}{n}+\frac{1}{m}-\frac{1}{N}\right)
\end{gathered}
$$

$$
f=f_{1}+\cdots+f_{I}
$$

$$
\rho=\frac{f_{1}}{f} \rho_{1}+\cdots+\frac{f_{I}}{f} \rho_{I}
$$

$$
\begin{aligned}
\omega_{2}= & -\frac{f}{4}\left(1-\frac{1}{\rho}\right)^{2}+\frac{1}{24} \\
& \cdot\left[f_{1}\left(f_{1}-1\right)+\cdots+f_{I}\left(f_{I}-1\right)\right]\left(\frac{1}{n^{2}}+\frac{1}{m^{2}}-\frac{1}{N^{2}}\right) \frac{1}{\rho^{2}} .
\end{aligned}
$$

Then the asymptotic distribution of the likelihood-ratio criterion is given by

$$
\begin{aligned}
& P\{-2 \rho \ln Q \leq z\} \simeq P\left\{\chi^{2}(f) \leq z\right\} \\
& \quad+\omega_{2}\left[P\left\{\chi^{2}(f+4) \leq z\right\}-P\left\{\chi^{2}(f) \leq z\right\}\right] .
\end{aligned}
$$

Proof: Omitted, straightforward but cumbersome calculations.

\section{REFERENCES}

[1] F. T. Ulaby, R. K. Moore, and A. K. Fung, Microwave Remote Sensing: Active and Passive. Norwood, MA: Artech House, 1986, vol. 3.

[2] H. Skriver, M. T. Svendsen, and A. G. Thomsen, "Multitemporal $L$ - and C-band polarimetric signatures of crops," IEEE Trans. Geosci. Remote Sensing, vol. 37, pp. 2413-2429, Sept. 1999.

[3] J. J. van Zyl and F. T. Ulaby, "Scattering matrix representation for simple targets," in Radar Polarimetry for Geoscience Applications, F. T. Ulaby and C. Elachi, Eds. Norwood, MA: Artech House, 1990.

[4] N. T. Goodman, "Statistical analysis based on a certain multivariate complex Gaussian distribution (An introduction)," Ann. Math. Stat., vol. 34, pp. 152-177, 1963.

[5] S. V. Nghiem, S. H. Yueh, R. Kwok, and F. K. Li, "Symmetry properties in polarimetric remote sensing," Radio Sci., vol. 27, no. 5, pp. 693-711, 1992.

[6] A. A. Nielsen, R. Larsen, and H. Skriver, "Change detection in bi-temporal EMISAR data from Kalø, Denmark, by means of canonical correlations analysis," in Proc. 3rd Int. Airborne Remote Sensing Conf. and Exhibition, Copenhagen, Denmark, July 7-10, 1997.

[7] A. A. Nielsen, "Change detection in multispectral bi-temporal spatial data using orthogonal transformations," in Proc. 8th Austral-Asian Sensing Conf., Canberra ACT, Australia, Mar. 25-29, 1996.

[8] A. A. Nielsen and K. Conradsen, "Multivariate alteration detection (MAD) in multispectral, bi-temporal image data: A new approach to change detection studies," Dept. Mathematical Modelling, Tech. Univ. Denmark, Lyngby, Denmark, Tech. Rep. 1997-11, 1997.

[9] A. A. Nielsen, K. Conradsen, and J. J. Simpson, "Multivariate alteration detection (MAD) and MAF post-processing in multispectral, bi-temporal image data: New approaches to change detection studies," Remote Sens. Environ., vol. 19, pp. 1-19, 1998.

[10] A. A. Nielsen, "Multi-channel remote sensing data and orthogonal transformations for change detection," in Machine Vision and Advanced Image Processing in Remote Sensing, I. Kanellopoulos, G. G. Wilkinson, and T. Moons, Eds. Berlin, Germany: Springer, 1999, pp. 37-48.

[11] J. Schou, H. Skriver, K. Conradsen, and A. A. Nielsen, "CFAR edge detector for polarimetric SAR images," IEEE Trans. Geosci. Remote Sensing, vol. 41, pp. 20-32, Jan. 2003.

[12] S. N. Madsen, E. L. Christensen, N. Skou, and J. Dall, "The Danish SAR system: Design and initial tests," IEEE Trans. Geosci. Remote Sensing, vol. 29, pp. 417-476, May 1991.

[13] E. L. Christensen, N. Skou, J. Dall, K. Woelders, J. H. Jørgensen, J. Granholm, and S. N. Madsen, "EMISAR: An absolutely calibrated polarimetric $L$ - and C-band SAR," IEEE Trans. Geosci. Remote Sensing, vol. 36, pp. 1852-1865, Nov. 1998. 
[14] R. Touzi, A. Lopes, and P. Bousquez, "A statistical and geometrical edge detector for SAR images," IEEE Trans. Geosci. Remote Sensing, vol. 26, pp. 764-773, Nov. 1988.

[15] A. Lopes, E. Nezry, R. Touzi, and H. Laur, "Structure detection and statistical adaptive speckle filtering in SAR images," Int. J. Remote Sens., vol. 13, no. 9, pp. 1735-1758, 1993.

[16] T. W. Anderson, An Introduction to Multivariate Statistical Analysis, 2nd ed. New York: Wiley, 1984.

[17] C. G. Khatri, "Classical statistical analysis based on a certain multivariate complex Gaussian distribution," Ann. Math. Stat., vol. 36, pp. 98-114, 1965.

[18] S. A. Andersson, H. K. Brøns, and S. T. Jensen, "Distribution of eigenvalues in multivariate statistical analysis," Inst. Mathematical Statistics, Univ. Copenhagen, Copenhagen, Denmark, 1982

[19] G. Gabrielsen, "Fordeling af egenværdier i relle, komplekse og kvaternion Wishart fordelinger," M.S. thesis (in Danish), Inst. Mathematical Statistics, Univ. Copenhagen, Copenhagen, Denmark, 1975.

[20] H. H. Andersen, M. Højbjerre, D. Sørensen, and P. S. Eriksen, Linear and Graphical Models for the Multivariate Complex Normal Distribution. Berlin, Germany: Springer-Verlag, 1995, vol. 101, Lecture Notes in Statistics.

[21] G. E. P. Box, "A general distribution theory for a class of likelihood criteria," Biometrika, vol. 36, pp. 317-346, 1949.

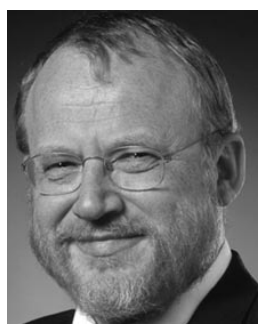

Knut Conradsen received the M.S. degree from the Department of Mathematics, University of Copenhagen, Copenhagen, Denmark, in 1970.

$\mathrm{He}$ is currently a Professor with the Informatics and Mathematical Modelling, Technical University of Denmark (DTU), Lyngby, Denmark. Since 1995, he has been Deputy Rector (or Vice President) of DTU. His main research interest is the application of statistics and statistical models to real-life problems. He has worked on many national and international projects on the application of statistical methods in a wide range of applications, recently mainly in remote sensing. He has also conducted extensive studies in the development and application of mathematical and statistical methods concerning spatial, multi/hyperspectral, and multitemporal data, including both optical and radar data.

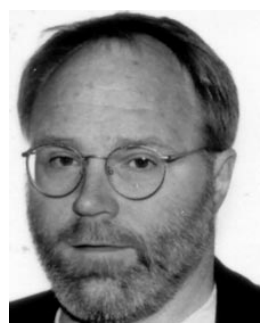

Allan Aasbjerg Nielsen received the M.S. degree from the Department of Electrophysics, Technical University of Denmark, Lyngby, Denmark, in 1978, and the Ph.D. degree from Informatics and Mathematical Modelling (IMM), Technical University of Denmark, in 1994.

$\mathrm{He}$ is currently an Associate Professor with IMM. He is currently working with IMM's Section for Geoinformatics. He has been with the Danish Defense Research Establishment from 1977 to 1978. He has worked on energy conservation in housing with the Thermal Insulation Laboratory, Technical University of Denmark, from 1978 to 1985 . Since 1985, he has been with the Section for Image Analysis, IMM. Since then, he has worked on several national and international projects on the development, implementation, and application of statistical methods and remote sensing in mineral exploration, mapping, geology, environment, oceanography, geodesy, and agriculture funded by industry, the European Union, Danida (the Danish International Development Agency), and the Danish National Research Councils.

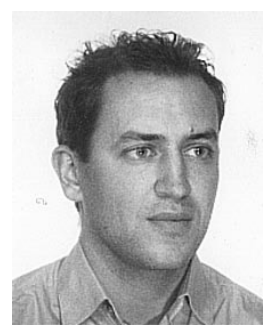

Jesper Schou received the M.S. degree in engineering and the Ph.D. degree in electrical engineering from the Technical University of Denmark, Lyngby, Denmark, in 1997 and 2001 respectively. His primary research interests during his Ph.D studies was image analysis and the processing of polarimetric SAR data, including filtering, structure detection, and segmentation.

He is currently working with 3-D scanner systems and related 3-D image analysis algorithms.

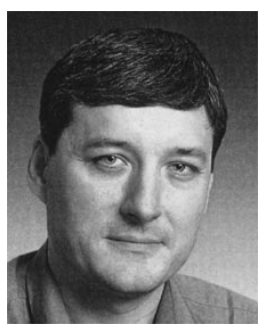

Henning Skriver received the M.S. degree and the Ph.D. degree from the Technical University of Denmark, Lyngby, in 1983 and 1989, respectively, both in electrical engineering.

He has been with the Section of Electromagnetic Systems (EMI), Department Ørsted, Technical University of Denmark (DTU), Lyngby, since 1983, where he is currently an Associate Professor. His work has been concerned primarily with various topics related to the utilization of SAR data for different applications. From 1983 to 1992, his main area of interest was retrieval of sea ice parameters from SAR data, including SAR data from ERS-1. Since 1992, he has covered different aspects of land applications of SAR data, such as forestry in the MAESTRO-1 project, and agricultural and environmental applications using both satellite SAR data and data from the Danish airborne polarimetric SAR, EMISAR. His interests also include various methods for processing of SAR data, such as SAR image simulation, SAR image filtering, speckle statistics, texture analysis, segmentation, calibration, and polarimetric analysis. He is currently a Project Manager for a project concerning the use of SAR data for cartographic mapping. 